\title{
GLOBAL ENERGETICS OF SOLAR FLARES. III. NONTHERMAL ENERGIES
}

\author{
Markus J. Aschwanden ${ }^{1}$, Gordon Holman ${ }^{2}$, Aidan O' Flannagain $^{3}$, Amir Caspi $^{4}$, James M. McTiernan $^{5}$, and \\ EDUARD P. KONTAR ${ }^{6}$ \\ ${ }^{1}$ Lockheed Martin, Solar and Astrophysics Laboratory, Org. A021S, Bldg. 252, 3251 Hanover Street, Palo Alto, CA 94304, USA; aschwanden@1msal.com \\ ${ }^{2}$ Code 671, NASA Goddard Space Flight Center, Greenbelt, MD 20771, USA; gordon.d.holman@nasa.gov \\ ${ }^{3}$ Astrophysics Research Group, School of Physics, Trinity College Dublin, Dublin 2, Ireland; aidanoflann@gmail.com \\ ${ }^{4}$ Planetary Science Directorate, Southwest Research Institute, Boulder, CO 80302, USA; amir.caspi@swri.org \\ ${ }^{5}$ Space Sciences Laboratory, University of California, Berkeley, CA 94720, USA; jimm@ssl.berkeley.edu \\ ${ }^{6}$ School of Physics and Astronomy, University of Glasgow, G12 8QQ, Glasgow, Scotland, UK; eduard.kontar@astro.gla.ac.uk \\ Received 2016 June 13; revised 2016 August 22; accepted 2016 August 23; published 2016 November 14
}

\begin{abstract}
This study entails the third part of a global flare energetics project, in which Ramaty High-Energy Solar Spectroscopic Imager (RHESSI) data of $191 \mathrm{M}$ and X-class flare events from the first 3.5 years of the Solar Dynamics Observatory mission are analyzed. We fit a thermal and a nonthermal component to RHESSI spectra, yielding the temperature of the differential emission measure (DEM) tail, the nonthermal power-law slope and flux, and the thermal/nonthermal cross-over energy $e_{\mathrm{co}}$. From these parameters, we calculate the total nonthermal energy $E_{\mathrm{nt}}$ in electrons with two different methods: (1) using the observed cross-over energy $e_{\mathrm{co}}$ as low-energy cutoff, and (2) using the low-energy cutoff $e_{\mathrm{wt}}$ predicted by the warm thick-target bremsstrahlung model of Kontar et al. Based on a mean temperature of $T_{e}=8.6 \mathrm{MK}$ in active regions, we find low-energy cutoff energies of $e_{\mathrm{wt}}=6.2 \pm 1.6 \mathrm{keV}$ for the warm-target model, which is significantly lower than the cross-over energies $e_{\mathrm{co}}=21 \pm 6 \mathrm{keV}$. Comparing with the statistics of magnetically dissipated energies $E_{\mathrm{mag}}$ and thermal energies $E_{\mathrm{th}}$ from the two previous studies, we find the following mean (logarithmic) energy ratios with the warm-target model: $E_{\mathrm{nt}}=0.41 E_{\mathrm{mag}}, E_{\mathrm{th}}=0.08 E_{\mathrm{mag}}$, and $E_{\mathrm{th}}=0.15 E_{\mathrm{nt}}$. The total dissipated magnetic energy exceeds the thermal energy in $95 \%$ and the nonthermal energy in $71 \%$ of the flare events, which confirms that magnetic reconnection processes are sufficient to explain flare energies. The nonthermal energy exceeds the thermal energy in $85 \%$ of the events, which largely confirms the warm thick-target model.
\end{abstract}

Key words: radiation mechanisms: nonthermal - Sun: flares - Sun: particle emission - Sun: X-rays, gamma rays

Supporting material: machine-readable table

\section{INTRODUCTION}

We undertake a systematic survey of the global energetics of solar flares and coronal mass ejections (CMEs) observed during the Solar Dynamics Observatory (SDO) era, which includes all $\mathrm{M}$ - and X-class flares during the first 3.5 years of the $S D O$ mission, covering some 400 flare events. This project embodies the most comprehensive survey about various forms of energies that can be detected during flares, such as the dissipated magnetic energy, the thermal energy, the nonthermal energy, the radiative and conductive energy, and the kinetic energy of associated CMEs. Two studies have been completed previously, containing statistics on magnetic energies (Aschwanden et al. 2014, Paper I), and thermal energies (Aschwanden et al. 2015, Paper II). In this study, we focus on the third part of this "global flare energetics project," which entails the statistics of nonthermal energies in hard X-ray-producing electrons that are observed in hard X-rays and gamma rays, using data from the Ramaty High-Energy Solar Spectroscopic Imager (RHESSI) spacecraft (Lin et al. 2002).

The quantitative measurement of nonthermal energies in solar flares allows us some tests of fundamental nature. One concept or working hypothesis is that all primary energy input in solar flares is provided by dissipation of free magnetic energy, for instance by a magnetic reconnection process, which supplies energy for secondary processes, such as for the acceleration of charged particles and heating of flare plasma. The accelerated (nonthermal) particles either escape from the flare site into interplanetary space, or more likely precipitate down to the chromosphere where they subsequently become thermalized and radiate in hard X-rays and gamma rays, according to the thicktarget bremsstrahlung model (Brown 1971). In this picture, we expect that the total nonthermal energy $E_{\mathrm{nt}}$ (in electrons and ions) produced in flares should not exceed the dissipated magnetic (free) energy $E_{\text {mag }}$, but on the other hand should yield an upper limit on the thermal energy $E_{\mathrm{th}}$ inferred from the soft X-ray and EUV-emitting plasma. Alternative mechanisms to the thick-target model envision thermal conduction fronts (e.g., Brown et al. 1979) or direct heating processes (e.g., Duijveman et al. 1981). In the previous two papers, we proved the inequality $E_{\mathrm{mag}}>E_{\mathrm{th}}$, for which we found an energy conversion ratio of $E_{\mathrm{th}} / E_{\mathrm{mag}} \approx 0.02-0.40$ (Paper II), which is about an order of magnitude higher than estimated in a previous statistical study (Emslie et al. 2012), where an ad hoc value (30\%) of the ratio of the free magnetic energy to the potential field energy was estimated. In this work, Paper III, we investigate the expected inequalities $E_{\mathrm{mag}}>E_{\mathrm{nt}}>E_{\mathrm{th}}$. If these two inequalities are not fulfilled, it could be attributed to insufficient accuracy of the energy measurements, or alternatively may question the correctness of the associated low-energy cutoff model, the applied magnetic reconnection models, or the efficiency of the electron thick-target bremsstrahlung model. Such an outcome would have important consequences in our understanding of solar flare models and the related predictability of the most extreme space weather events.

The measurement of nonthermal energies in solar flares requires a spectral fit of the hard X-ray spectrum in the energy range of $\varepsilon \approx 10-30 \mathrm{keV}$ (Aschwanden 2007), from spectral 
data as they are available from the HXRBS/SMM, BATSE/ CGRO, or RHESSI instruments. Since the total nonthermal energy contained in a flare requires integrations over the temporal and spectral range, the largest uncertainty of this quantity comes from the assumed low-energy cutoff because it cannot be directly measured due to the strong thermal component that often dominates the spectrum at $\varepsilon \lesssim 20 \mathrm{keV}$ during solar flares (for a review, see Holman et al. 2011). In a few cases, low-energy cutoffs of the nonthermal spectrum could be determined by regularized inversion methods at $e_{c}=20-40 \mathrm{keV}$ (Kasparova et al. 2005), $e_{c} \approx 20 \mathrm{keV}$ (Kontar $\&$ Brown 2006), and $e_{c}=13-19 \mathrm{keV}$ (Kontar et al. 2008). For the 2002 July 23 flare, Holman (2003) deduced upper limits to low-energy cutoffs by determining the highest values consistent with acceptable spectral fits. Sui et al. (2007) deduced the lowenergy cutoff in a flare from the combination of spectral fits and the time evolution of the X-ray emission in multiple energy bands. Sui et al. (2007) deduced low-energy cutoffs for several flares with relatively weak thermal components ("early impulsive flares") from spectral fits, with values ranging from $15-50 \mathrm{keV}$. In the late peak of a multi-peaked flare, Warmuth et al. (2009) inferred low-energy cutoff values exceeding $100 \mathrm{keV}$, but this unusually high value could possibly also be explained by high-energy electrons that accumulate by trapping after the flare peak (Aschwanden et al. 1997). Using a novel method of differentiating nonthermal electrons by their time-offlight delay from thermal electrons by their thermal conduction time delay, a thermal-nonthermal cross-over energy of $e_{c}=$ $18.0 \pm 3.4 \mathrm{keV}$ (or a range of $e_{c}=10-28 \mathrm{keV}$ ) was established for the majority $(68 \%)$ of 65 analyzed flare events (Aschwanden 2007).

Statistical measurements of nonthermal flare energies have been calculated from HXRBS/SMM data (Crosby et al. 1993), or from RHESSI data (Hannah et al. 2008; Christe et al. 2008; Emslie et al. 2012). The low-energy cutoff was taken into account by assuming a fixed energy cutoff of $e_{c}=25 \mathrm{keV}$ (Crosby et al. 1993), a fixed spectral slope of $\gamma=-1.5$ below the thermal-nonthermal cross-over energy $e_{\mathrm{co}}$ (Hannah et al. 2008), or by adopting the largest energy $e_{c}$ that still produces a goodness-of-fit with $\chi^{2} \approx 1$ for the nonthermal power-law fit (Emslie et al. 2012). Low-energy cutoffs for microflares were estimated in the range of $e_{c} \approx 9-16 \mathrm{keV}$, with a median of $12 \mathrm{keV}$ (Hannah et al. 2008), using a numerical integration code of Holman (2003). The statistical study of Emslie et al. (2012) provides a comparison between nonthermal energies $E_{\mathrm{nt}}$, thermal energies $E_{\mathrm{th}}$, and dissipated magnetic energies $E_{\mathrm{mag}}$, yielding mean (logarithmic) ratios of $E_{\mathrm{th}} \approx 0.005 E_{\mathrm{mag}}$ and $E_{\mathrm{nt}} \approx 0.03 E_{\mathrm{mag}}$. These results conform to the expected inequalities, but the magnetic energies $E_{\mathrm{mag}}$ were actually not measured in the study of Emslie et al. (2012), and most likely were overestimated by an order of magnitude (Paper I). The dissipated magnetic energies $E_{\text {mag }}$ were for the first time quantitatively measured in Paper I, by automated tracing of coronal flare loops from AIA/SDO images and by forward-fitting of a nonlinear force-free magnetic field (NLFFF) model based on the vertical-current approximation (Aschwanden 2013, 2016).

The content of this paper consists of a theoretical model to estimate the low-energy cutoff and the nonthermal energy (Section 2), a description of the data analysis method (Section 3), the results of the data analysis of $191 \mathrm{M}$ and X-class flare events observed with RHESSI (Section 4), a discussion of the results (Section 5), and conclusions (Section 6).

\section{THEORY}

\subsection{Nonthermal Energy in Electrons}

The nonthermal energy in flare electrons is generally calculated with the thick-target model (Brown 1971), which expresses the hard X-ray photon spectrum by a convolution of the electron injection spectrum with the Bethe-Heitler bremsstrahlung cross-section. According to this model, the observed hard X-ray photon spectrum $I(\varepsilon)$ observed at Earth can be approximated by a power-law function with a slope $\gamma$ for the nonthermal energies, while the spectral index generally changes at the lower (thermal) energies. Thus, the nonthermal spectrum is defined as (e.g., see the textbook by Aschwanden 2004, chapter 13),

$$
I(\varepsilon)=A \varepsilon^{-\gamma} \quad\left(\text { photons } \mathrm{cm}^{-2} \mathrm{~s}^{-1} \mathrm{keV}^{-1}\right),
$$

which yields a thick-target (nonthermal) electron injection spectrum $f_{e}(e)$,

$$
f_{e}(e)=2.68 \times 10^{33} b(\gamma) A e^{-(\gamma+1)} \quad\left(\text { electrons } \mathrm{keV}^{-1} \mathrm{~s}^{-1}\right),
$$

which is a power-law function also, but with a slope $\delta=\gamma+1$ that is steeper by one, and $b(\gamma)$ is an auxiliary function related to the beta function. The detailed shape of a nonthermal electron spectrum that is affected by a low-energy cutoff is simulated in Holman (2003), showing a gradual flattening at lower energies. Note that we use the symbol $\varepsilon$ for photon energies, while we use the symbol $e$ for electron energies. The total power in nonthermal electrons above some cutoff energy $e_{c}$, i.e., $P\left(e \geqslant e_{c}\right)$, is

$$
P\left(e \geqslant e_{c}\right)=4.3 \times 10^{24} \frac{b(\gamma)}{(\gamma-1)} A\left(e_{c}\right)^{-(\gamma-1)} \quad\left(\mathrm{erg} \mathrm{s}^{-1}\right) .
$$

Thus, the three observables of the photon flux $A$, the photon power-law slope $\gamma$, and the low-energy cutoff energy $e_{c}$ are required to calculate the power during a selected flare time interval, which can be calculated with the OSPEX package of the SolarSoftWare library of the Interactive Data Language software (see RHESSI webpage http://hesperia.gsfc.nasa.gov/ ssw/packages/spex/doc/ospex_explanation.html).

In order to calculate the total nonthermal energy $E_{\mathrm{nt}}$ during an entire flare, we have to integrate the power as a function of time,

$$
E_{\mathrm{nt}}=\int_{t_{\mathrm{start}}}^{t_{\mathrm{end}}} P\left(e>e_{c}(t), t\right) d t \quad(\mathrm{erg}) .
$$

While the photon fluxes $A(t)$ and the spectral slopes $\gamma(t)$ can readily be measured from a time series of hard X-ray photon spectra (Equation (1)), the largest uncertainty in the determination of the nonthermal energy is the low-energy cutoff energy $e_{c}(t)$ between the thermal and nonthermal hard X-ray components, typically expected in the range of $\approx 10-30 \mathrm{keV}$ (see Table 3 in Aschwanden 2007). In the following, we outline two different theoretical models of the low-energy cutoff that are applied in this study. 
Table 1

Nonthermal Energy Parameters Derived in 191 Flare Events Observed with RHESSI

\begin{tabular}{|c|c|c|c|c|c|c|c|c|c|c|c|}
\hline \# & $\begin{array}{c}\text { Flare } \\
\text { Start Time }\end{array}$ & $\begin{array}{l}G O E S \\
\text { Class }\end{array}$ & $\begin{array}{l}\text { Helio- } \\
\text { Graphic } \\
\text { position }\end{array}$ & $\begin{array}{c}\text { Flare } \\
\text { Duration } \\
d \\
(\mathrm{~s})\end{array}$ & $\begin{array}{c}\text { Peak } \\
\text { Counts } \\
P \\
\left(\text { cts s }^{-1}\right)\end{array}$ & $\begin{array}{c}\text { Total } \\
\text { Counts } \\
C \\
\text { (cts) }\end{array}$ & $\begin{array}{l}\text { Fitted } \\
\text { Energy } \\
\text { range } \\
(\mathrm{keV})\end{array}$ & $\begin{array}{c}\text { Cutoff } \\
\text { Energy } \\
e_{\mathrm{wt}} \\
(\mathrm{keV})\end{array}$ & $\begin{array}{c}\text { Nonthermal } \\
\text { Energy } \\
E_{\mathrm{wt}} \\
(\mathrm{erg})\end{array}$ & $\begin{array}{c}\text { Energy } \\
\text { Ratio } \\
E_{\mathrm{th}} / E_{\mathrm{wt}}\end{array}$ & $\begin{array}{c}\text { Energy } \\
\text { Ratio } \\
E_{\mathrm{wt}} / E_{\text {mag }}\end{array}$ \\
\hline 1 & 201006120030 & M2.0 & N23W47 & 904 & 92 & $1.3 \mathrm{E}+05$ & {$[8-20]$} & 2.6 & $1.0 \mathrm{E}+30$ & $6.98^{*}$ & $\ldots$ \\
\hline 2 & 201006130530 & M1.0 & S24W82 & 1852 & 688 & $1.3 \mathrm{E}+06$ & [6-20] & 4.9 & $5.4 \mathrm{E}+28$ & $75.95^{*}$ & $\ldots$ \\
\hline 4 & 201010161907 & M2.9 & S18W26 & 1572 & 3312 & $3.1 \mathrm{E}+06$ & {$[6-26]$} & 5.6 & $6.2 \mathrm{E}+31$ & 0.31 & 2.21 \\
\hline 6 & 201011051243 & M1.0 & S20E75 & 2980 & 400 & $2.2 \mathrm{E}+06$ & [6-20] & 7.1 & $6.5 \mathrm{E}+31$ & 0.12 & $\ldots$ \\
\hline 8 & 201101280044 & M1.3 & N16W88 & 1760 & 1968 & $4.8 \mathrm{E}+06$ & [6-20] & 7.2 & $3.9 \mathrm{E}+31$ & $\ldots$ & $\ldots$ \\
\hline 10 & 201102131728 & M6.6 & S21E04 & 2324 & 6384 & $2.5 \mathrm{E}+07$ & {$[8-30]$} & 8.3 & $9.3 \mathrm{E}+32$ & $0.022^{*}$ & 10.98 \\
\hline 12 & 201102150144 & $\mathrm{X} 2.2$ & S21W12 & 2628 & 26868 & $9.8 \mathrm{E}+07$ & {$[10-50]$} & 5.8 & $1.1 \mathrm{E}+33$ & 0.073 & 9.34 \\
\hline 13 & 201102160132 & M1.0 & S22W27 & 1368 & 1072 & $1.5 \mathrm{E}+06$ & {$[8-40]$} & 6.8 & $4.4 \mathrm{E}+31$ & 0.17 & 0.39 \\
\hline 15 & 201102161419 & M1.6 & S23W33 & 1692 & 1039 & $1.3 \mathrm{E}+06$ & {$[6-30]$} & 6.9 & $3.8 \mathrm{E}+31$ & 0.17 & 0.21 \\
\hline 16 & 201102180955 & M6.6 & S21W55 & 1780 & 6082 & $6.5 \mathrm{E}+06$ & {$[6-30]$} & 6.3 & $5.3 \mathrm{E}+32$ & 0.0080 & 38.49 \\
\hline 18 & 201102181259 & M1.4 & S20W70 & 1944 & 1904 & $3.6 \mathrm{E}+06$ & [6-30] & 6.1 & $2.4 \mathrm{E}+31$ & 0.088 & $\ldots$ \\
\hline 19 & 201102181400 & M1.0 & N17E04 & 1264 & 432 & $6.4 \mathrm{E}+05$ & [8-20] & 6.8 & $1.0 \mathrm{E}+31$ & 0.49 & 0.39 \\
\hline 20 & 201102182056 & M1.3 & N15E00 & 884 & 1200 & $2.2 \mathrm{E}+06$ & {$[6-30]$} & 7.0 & $4.7 \mathrm{E}+31$ & 0.095 & 3.11 \\
\hline 21 & 201102240723 & M3.5 & N14E87 & 3332 & 2032 & $5.0 \mathrm{E}+06$ & {$[8-30]$} & 4.8 & $2.9 \mathrm{E}+31$ & 0.58 & $\ldots$ \\
\hline 22 & 201102281238 & M1.1 & N22E35 & 732 & 688 & $1.2 \mathrm{E}+06$ & [10-30] & 6.3 & $8.6 \mathrm{E}+31$ & 0.074 & 2.88 \\
\hline 23 & 201103070500 & M1.2 & N23W47 & 1340 & 880 & $1.5 \mathrm{E}+06$ & {$[6-30]$} & 7.4 & $2.4 \mathrm{E}+31$ & 0.019 & $\ldots$ \\
\hline 26 & 201103070914 & M1.8 & N27W46 & 348 & 1776 & $1.6 \mathrm{E}+06$ & [6-30] & 4.4 & $1.8 \mathrm{E}+31$ & 0.0093 & $\ldots$ \\
\hline 28 & 201103071943 & M3.7 & N30W48 & 3196 & 1328 & $6.7 \mathrm{E}+06$ & [10-30] & 3.4 & $1.8 \mathrm{E}+31$ & 1.30 & $\cdots$ \\
\hline 29 & 201103072145 & M1.5 & S17W82 & 1232 & 720 & $1.0 \mathrm{E}+06$ & [8-30] & 6.4 & $4.0 \mathrm{E}+31$ & 0.038 & $\ldots$ \\
\hline 30 & 201103080224 & M1.3 & S18W80 & 1460 & 752 & $6.8 \mathrm{E}+05$ & [6-30] & 7.3 & $3.2 \mathrm{E}+31$ & 0.088 & $\ldots$ \\
\hline 31 & 201103080337 & M1.5 & S21E72 & 2768 & 108 & $6.5 \mathrm{E}+05$ & [12-30] & 3.9 & $2.8 \mathrm{E}+30$ & 4.80 & $\ldots$ \\
\hline 33 & 201103081808 & M4.4 & S17W88 & 848 & 1712 & $5.6 \mathrm{E}+06$ & {$[8-30]$} & 6.3 & $7.8 \mathrm{E}+32$ & 0.020 & $\ldots$ \\
\hline 34 & 201103081946 & M1.5 & S19W87 & 6044 & 176 & $1.3 \mathrm{E}+06$ & [6-20] & 6.3 & $3.7 \mathrm{E}+31$ & 0.17 & $\ldots$ \\
\hline 37 & 201103092313 & $\mathrm{X} 1.5$ & N10W11 & 1660 & 4938 & $8.3 \mathrm{E}+06$ & {$[10-40]$} & 5.8 & $1.1 \mathrm{E}+33$ & 0.074 & 4.25 \\
\hline 38 & 201103102234 & M1.1 & S25W86 & 1588 & 192 & $3.1 \mathrm{E}+05$ & {$[8-30]$} & 6.7 & $4.5 \mathrm{E}+31$ & 0.016 & 0.16 \\
\hline 40 & 201103141930 & M4.2 & N16W49 & 2308 & 2988 & $3.3 \mathrm{E}+06$ & [8-30] & 8.2 & $4.1 \mathrm{E}+32$ & 0.021 & $\ldots$ \\
\hline 41 & 201103150018 & M1.0 & N11W83 & 1500 & 1648 & $7.1 \mathrm{E}+05$ & {$[8-30]$} & 5.0 & $4.6 \mathrm{E}+30$ & 0.077 & $\ldots$ \\
\hline 46 & 201104220435 & M1.8 & S19E40 & 3124 & 880 & $3.5 \mathrm{E}+06$ & [10-30] & 6.7 & $1.1 \mathrm{E}+32$ & 0.098 & 2.47 \\
\hline 48 & 201105282109 & M1.1 & S21E70 & 2848 & 624 & $2.1 \mathrm{E}+06$ & [6-30] & 7.3 & $1.4 \mathrm{E}+31$ & 0.39 & $\ldots$ \\
\hline 49 & 201105291008 & M1.4 & S20E64 & 3552 & 448 & $3.5 \mathrm{E}+06$ & [7-25] & 6.5 & $5.3 \mathrm{E}+31$ & 0.15 & $\ldots$ \\
\hline 50 & 201106070616 & M2.5 & S22W53 & 3608 & 944 & $5.1 \mathrm{E}+06$ & {$[8-30]$} & 3.3 & $1.4 \mathrm{E}+31$ & 1.92 & $\ldots$ \\
\hline 51 & 201106142136 & M1.3 & N14E77 & 2356 & 688 & $1.7 \mathrm{E}+06$ & [6-30] & 5.3 & $5.5 \mathrm{E}+31$ & 0.13 & $\ldots$ \\
\hline 52 & 201107271548 & M1.1 & $\mathrm{N} 20 \mathrm{E} 41$ & 2004 & 256 & $4.6 \mathrm{E}+05$ & {$[6-30]$} & 6.8 & $6.4 \mathrm{E}+30$ & 1.86 & 0.20 \\
\hline 53 & 201107300204 & M9.3 & N16E35 & 1460 & 6115 & $6.5 \mathrm{E}+06$ & {$[8-30]$} & 6.9 & $1.0 \mathrm{E}+33$ & 0.028 & 11.05 \\
\hline 54 & 201108020519 & M1.4 & N16W11 & 6208 & 1895 & $3.3 \mathrm{E}+06$ & [10-30] & 5.3 & $1.1 \mathrm{E}+31$ & 0.97 & 0.096 \\
\hline 55 & 201108030308 & M1.1 & N15W23 & 2760 & 944 & $2.4 \mathrm{E}+06$ & {$[6-30]$} & 6.9 & $3.4 \mathrm{E}+31$ & 0.12 & 1.61 \\
\hline 56 & 201108030429 & M1.7 & N16E10 & 1268 & 2160 & $1.6 \mathrm{E}+06$ & {$[8-30]$} & 6.0 & $3.2 \mathrm{E}+31$ & 0.098 & 0.14 \\
\hline 61 & 201108090748 & X6.9 & N20W69 & 2256 & 53158 & $7.3 \mathrm{E}+07$ & [12-40] & 5.5 & $3.2 \mathrm{E}+33$ & 0.041 & $\ldots$ \\
\hline 63 & 201109050408 & M1.6 & N18W87 & 1516 & 624 & $2.3 \mathrm{E}+06$ & {$[6-30]$} & 6.7 & $1.5 \mathrm{E}+31$ & 0.18 & $\ldots$ \\
\hline 64 & 201109050727 & M1.2 & N18W87 & 2464 & 624 & $2.0 \mathrm{E}+06$ & [10-25] & 11.7 & $3.5 \mathrm{E}+29$ & 3.44 & $\ldots$ \\
\hline 65 & 201109060135 & M5.3 & N15W03 & 692 & 4724 & $3.9 \mathrm{E}+06$ & {$[10-40]$} & 6.8 & $3.2 \mathrm{E}+32$ & 0.069 & 2.86 \\
\hline 66 & 201109062212 & $\mathrm{X} 2.1$ & N16W15 & 1024 & 21072 & $2.3 \mathrm{E}+07$ & [12-40] & 5.0 & $7.6 \mathrm{E}+31$ & 0.68 & 0.41 \\
\hline 68 & 201109081532 & M6.7 & N17W39 & 1764 & 2439 & $4.7 \mathrm{E}+06$ & {$[8-25]$} & 7.3 & $1.5 \mathrm{E}+33$ & 0.019 & 10.99 \\
\hline 69 & 201109090601 & M2.7 & N14W48 & 1644 & 3824 & $6.3 \mathrm{E}+06$ & {$[10-40]$} & 5.2 & $8.9 \mathrm{E}+31$ & 0.20 & $\ldots$ \\
\hline 70 & 201109091239 & M1.2 & N15W50 & 408 & 96 & $1.0 \mathrm{E}+05$ & {$[7-30]$} & 5.8 & $9.2 \mathrm{E}+30$ & 0.41 & $\ldots$ \\
\hline 71 & 201109100718 & M1.1 & N14W64 & 2488 & 688 & $3.0 \mathrm{E}+06$ & [10-30] & 7.3 & $4.1 \mathrm{E}+31$ & 0.14 & $\ldots$ \\
\hline 73 & 201109220953 & M1.1 & N24W55 & 1508 & 624 & $1.3 \mathrm{E}+06$ & {$[9-30]$} & 8.2 & $4.2 \mathrm{E}+31$ & 0.084 & $\ldots$ \\
\hline 75 & 201109230147 & M1.6 & N24W64 & 1832 & 624 & $2.1 \mathrm{E}+06$ & [10-30] & 8.7 & $4.0 \mathrm{E}+31$ & 0.093 & $\ldots$ \\
\hline 76 & 201109232154 & M1.6 & N12E56 & 2456 & 5616 & $2.2 \mathrm{E}+06$ & [10-30] & 8.3 & $4.7 \mathrm{E}+31$ & 0.14 & $\cdots$ \\
\hline 77 & 201109232348 & M1.9 & N12E56 & 2020 & 1008 & $2.7 \mathrm{E}+06$ & {$[8-30]$} & 5.6 & $7.1 \mathrm{E}+31$ & 0.20 & $\ldots$ \\
\hline 78 & 201109240921 & $\mathrm{X} 1.9$ & N13E61 & 3008 & 18653 & $4.4 \mathrm{E}+07$ & {$[8-50]$} & 7.4 & $8.2 E+33$ & 0.0027 & $\ldots$ \\
\hline 81 & 201109241719 & M3.1 & N13E54 & 1324 & 2160 & $3.3 \mathrm{E}+06$ & [6-30] & 5.2 & $1.2 \mathrm{E}+32$ & 0.028 & $\cdots$ \\
\hline 83 & 201109241909 & M3.0 & N15E50 & 1068 & 1520 & $4.0 \mathrm{E}+06$ & [7-30] & 5.4 & $1.1 \mathrm{E}+32$ & 0.22 & $\ldots$ \\
\hline 84 & 201109242029 & M5.8 & N13E52 & 1180 & 5051 & $8.1 \mathrm{E}+06$ & {$[8-40]$} & 6.7 & $2.1 \mathrm{E}+32$ & 0.042 & $\ldots$ \\
\hline 86 & 201109242345 & M1.0 & S28W66 & 2596 & 336 & $1.3 \mathrm{E}+06$ & [10-30] & 4.3 & $2.9 \mathrm{E}+30$ & 0.53 & $\ldots$ \\
\hline 88 & 201109250431 & M7.4 & N13E50 & 3640 & 5462 & $2.7 \mathrm{E}+07$ & {$[9-30]$} & 6.9 & $2.1 \mathrm{E}+33$ & 0.018 & $\ldots$ \\
\hline 90 & 201109250925 & M1.5 & S28W71 & 2720 & 656 & $2.7 \mathrm{E}+06$ & [7-30] & 6.9 & $5.2 \mathrm{E}+31$ & 0.074 & $\ldots$ \\
\hline 91 & 201109251526 & M3.7 & N15E39 & 676 & 1840 & $2.5 \mathrm{E}+06$ & {$[7-30]$} & 6.5 & $2.7 \mathrm{E}+31$ & 0.64 & 0.058 \\
\hline 93 & 201109260506 & M4.0 & N15E35 & 572 & 1957 & $2.5 \mathrm{E}+06$ & [10-30] & 7.4 & $3.6 \mathrm{E}+32$ & 0.032 & 0.51 \\
\hline 98 & 201110020037 & M3.9 & N10W13 & 3696 & 4336 & $9.4 \mathrm{E}+06$ & {$[10-30]$} & 6.9 & $4.2 \mathrm{E}+32$ & 0.044 & 6.62 \\
\hline 100 & 201110200310 & M1.6 & N18W88 & 1044 & 1392 & $3.5 \mathrm{E}+06$ & [10-30] & 7.2 & $1.5 \mathrm{E}+32$ & 0.012 & $\ldots$ \\
\hline
\end{tabular}


Table 1

(Continued)

\begin{tabular}{|c|c|c|c|c|c|c|c|c|c|c|c|}
\hline \# & $\begin{array}{c}\text { Flare } \\
\text { Start Time }\end{array}$ & $\begin{array}{c}G O E S \\
\text { Class }\end{array}$ & $\begin{array}{l}\text { Helio- } \\
\text { Graphic } \\
\text { position }\end{array}$ & $\begin{array}{c}\text { Flare } \\
\text { Duration } \\
d \\
(\mathrm{~s})\end{array}$ & $\begin{array}{c}\text { Peak } \\
\text { Counts } \\
P \\
\text { (cts s }^{-1} \text { ) }\end{array}$ & $\begin{array}{c}\text { Total } \\
\text { Counts } \\
C \\
\text { (cts) }\end{array}$ & $\begin{array}{c}\text { Fitted } \\
\text { Energy } \\
\text { range } \\
(\mathrm{keV})\end{array}$ & $\begin{array}{c}\text { Cutoff } \\
\text { Energy } \\
e_{\mathrm{wt}} \\
(\mathrm{keV})\end{array}$ & $\begin{array}{c}\text { Nonthermal } \\
\text { Energy } \\
E_{\mathrm{wt}} \\
(\mathrm{erg})\end{array}$ & $\begin{array}{c}\text { Energy } \\
\text { Ratio } \\
E_{\mathrm{th}} / E_{\mathrm{wt}}\end{array}$ & $\begin{array}{c}\text { Energy } \\
\text { Ratio } \\
E_{\mathrm{wt}} / E_{\mathrm{mag}}\end{array}$ \\
\hline 101 & 201110211253 & M1.3 & N05W79 & 760 & 624 & $9.9 \mathrm{E}+05$ & {$[6-30]$} & 5.3 & $9.3 \mathrm{E}+30$ & 0.28 & $\ldots$ \\
\hline 103 & 201110311455 & M1.1 & N20E88 & 3980 & 1392 & $3.8 \mathrm{E}+06$ & [10-30] & 6.7 & $1.3 \mathrm{E}+32$ & 0.0070 & $\ldots$ \\
\hline 110 & 201111050308 & M3.7 & N20E47 & 3752 & 1136 & $9.1 \mathrm{E}+06$ & [10-30] & 7.9 & $1.0 \mathrm{E}+32$ & 0.13 & $\ldots$ \\
\hline 111 & 201111051110 & M1.1 & N22E43 & 2392 & 320 & $9.7 \mathrm{E}+05$ & [10-30] & 6.9 & $1.3 \mathrm{E}+31$ & 0.25 & 0.044 \\
\hline 116 & 201111150903 & M1.2 & N21W72 & 2448 & 656 & $1.6 \mathrm{E}+06$ & {$[8-30]$} & 6.2 & $2.3 \mathrm{E}+31$ & 0.12 & $\ldots$ \\
\hline 120 & 201112260213 & M1.5 & S18W34 & 2812 & 624 & $1.4 \mathrm{E}+06$ & {$[10-30]$} & 5.6 & $6.8 \mathrm{E}+30$ & 1.21 & 0.72 \\
\hline 121 & 201112262012 & M2.3 & S18W44 & 1512 & 1456 & $3.2 \mathrm{E}+06$ & {$[10-30]$} & 6.7 & $1.0 \mathrm{E}+32$ & $\ldots$ & 3.98 \\
\hline 122 & 201112291340 & M1.9 & S25E70 & 2368 & 848 & $1.6 \mathrm{E}+06$ & {$[10-30]$} & 7.4 & $2.9 \mathrm{E}+31$ & 0.35 & $\ldots$ \\
\hline 123 & 201112292143 & M2.0 & S25E67 & 632 & 1008 & $1.2 \mathrm{E}+06$ & {$[10-30]$} & 7.7 & $8.4 \mathrm{E}+31$ & 0.079 & $\ldots$ \\
\hline 125 & 201112311309 & M2.4 & S25E46 & 1892 & 1584 & $1.6 \mathrm{E}+06$ & {$[10-30]$} & 6.7 & $8.3 E+31$ & 0.049 & $\ldots$ \\
\hline 126 & 201112311616 & M1.5 & $\mathrm{S} 22 \mathrm{E} 42$ & 1272 & 656 & $9.2 \mathrm{E}+05$ & {$[10-30]$} & 7.1 & $4.6 \mathrm{E}+31$ & 0.18 & 0.28 \\
\hline 154 & 201203172032 & M1.3 & S25W28 & 1236 & 1136 & $8.2 \mathrm{E}+05$ & [10-25] & 7.3 & $1.8 \mathrm{E}+31$ & 0.35 & 0.65 \\
\hline 156 & 201204161724 & M1.7 & N14E88 & 1932 & 352 & $1.5 \mathrm{E}+06$ & [10-20] & 7.5 & $4.0 \mathrm{E}+31$ & 0.37 & $\ldots$ \\
\hline 157 & 201204270815 & M1.0 & N13W26 & 732 & 528 & $6.4 \mathrm{E}+05$ & {$[10-30]$} & 6.2 & $2.1 \mathrm{E}+31$ & 0.34 & 4.72 \\
\hline 158 & 201205051319 & M1.4 & N11E78 & 200 & 560 & $1.4 \mathrm{E}+05$ & [10-30] & 1.6 & $5.5 \mathrm{E}+30$ & $0.71^{*}$ & $\ldots$ \\
\hline 159 & 201205052256 & M1.3 & N11E73 & 624 & 1200 & $9.6 \mathrm{E}+05$ & {$[10-30]$} & 5.8 & $3.8 \mathrm{E}+31$ & 0.091 & $\ldots$ \\
\hline 160 & 201205060112 & M1.1 & N11E73 & 1684 & 976 & $6.7 \mathrm{E}+05$ & {$[10-30]$} & 5.7 & $1.2 \mathrm{E}+31$ & 0.16 & $\ldots$ \\
\hline 163 & 201205081302 & M1.4 & N13E46 & 432 & 1264 & $1.1 \mathrm{E}+06$ & [10-30] & 4.9 & $1.9 \mathrm{E}+31$ & 0.25 & $\ldots$ \\
\hline 167 & 201205100411 & M5.7 & N12E19 & 1128 & 3339 & $5.9 \mathrm{E}+06$ & [10-30] & 3.1 & $2.5 \mathrm{E}+30$ & 7.59 & 0.017 \\
\hline 168 & 201205102020 & M1.7 & N12E10 & 1612 & 1712 & $2.3 \mathrm{E}+06$ & {$[10-30]$} & 5.4 & $6.4 \mathrm{E}+31$ & 0.17 & 0.50 \\
\hline 169 & 201205170125 & M5.1 & N07W88 & 2708 & 2416 & $1.3 \mathrm{E}+07$ & [10-30] & 4.7 & $4.1 \mathrm{E}+31$ & 0.96 & $\ldots$ \\
\hline 170 & 201206031748 & M3.3 & N15E33 & 852 & 1648 & $1.3 \mathrm{E}+06$ & {$[10-30]$} & 4.2 & $9.0 \mathrm{E}+29$ & 25.04 & 0.020 \\
\hline 173 & 201206091645 & M1.8 & S16E76 & 1724 & 1264 & $1.8 \mathrm{E}+06$ & {$[10-30]$} & 6.7 & $6.6 \mathrm{E}+31$ & 0.047 & $\ldots$ \\
\hline 176 & 201206141252 & M1.9 & S19E06 & 9628 & 880 & $4.3 \mathrm{E}+06$ & {$[10-30]$} & 3.8 & $2.6 \mathrm{E}+30$ & $1.05^{*}$ & 0.008 \\
\hline 178 & 201206290913 & M2.2 & N15E37 & 696 & 2160 & $1.2 \mathrm{E}+06$ & [10-30] & 6.5 & $2.3 \mathrm{E}+31$ & 0.16 & 0.23 \\
\hline 182 & 201207020026 & M1.1 & N15E01 & 1356 & 944 & $1.1 \mathrm{E}+06$ & [10-30] & 6.4 & $1.5 \mathrm{E}+31$ & 0.29 & 0.23 \\
\hline 187 & 201207040947 & M5.3 & S17W18 & 2416 & 8339 & $9.5 \mathrm{E}+06$ & {$[10-30]$} & 6.3 & $3.7 \mathrm{E}+32$ & 0.020 & 2.24 \\
\hline 189 & 201207041435 & M1.3 & S18W20 & 428 & 320 & $2.7 \mathrm{E}+05$ & [10-25] & 2.5 & $2.6 \mathrm{E}+29$ & 11.36 & 0.005 \\
\hline 190 & 201207041633 & M1.8 & N14W33 & 828 & 192 & $3.6 \mathrm{E}+05$ & [10-25] & 3.2 & $4.8 \mathrm{E}+29$ & 35.19 & 0.017 \\
\hline 195 & 201207050325 & M4.7 & S18W29 & 1768 & 4447 & $8.0 \mathrm{E}+06$ & [10-30] & 6.6 & $3.5 \mathrm{E}+32$ & 0.017 & 2.09 \\
\hline 196 & 201207050649 & M1.1 & S17W29 & 1208 & 912 & $2.5 \mathrm{E}+06$ & {$[10-30]$} & 6.7 & $5.3 \mathrm{E}+31$ & 0.068 & 0.42 \\
\hline 199 & 201207051139 & M6.1 & S18W32 & 1056 & 1536 & $1.9 \mathrm{E}+06$ & [10-30] & 4.4 & $1.8 \mathrm{E}+31$ & 1.12 & 0.14 \\
\hline 200 & 201207051305 & M1.2 & S18W36 & 1400 & 80 & $2.8 \mathrm{E}+05$ & [10-20] & 1.6 & $2.9 \mathrm{E}+29$ & 30.20 & 0.002 \\
\hline 203 & 201207060137 & M2.9 & S18W43 & 2748 & 4300 & $3.7 \mathrm{E}+06$ & {$[10-30]$} & 5.2 & $3.8 \mathrm{E}+31$ & 0.11 & 0.53 \\
\hline 205 & 201207060817 & M1.5 & S12W48 & 1392 & 1392 & $1.8 \mathrm{E}+06$ & {$[10-30]$} & 6.4 & $4.3 \mathrm{E}+31$ & 0.060 & $\ldots$ \\
\hline 208 & 201207061848 & M1.3 & S15E88 & 1348 & 256 & $4.0 \mathrm{E}+05$ & {$[10-30]$} & 5.7 & $3.2 \mathrm{E}+31$ & 0.12 & $\ldots$ \\
\hline 210 & 201207070310 & M1.2 & S17W55 & 1664 & 1200 & $1.7 \mathrm{E}+06$ & [10-30] & 6.6 & $5.5 \mathrm{E}+31$ & 0.062 & $\ldots$ \\
\hline 211 & 201207070818 & M1.0 & S16E76 & 684 & 400 & $8.1 \mathrm{E}+05$ & [10-30] & 4.8 & $5.1 \mathrm{E}+29$ & 2.97 & $\ldots$ \\
\hline 212 & 201207071057 & M2.6 & S17W59 & 520 & 3065 & $3.5 \mathrm{E}+06$ & {$[10-30]$} & 5.4 & $2.0 \mathrm{E}+32$ & 0.025 & $\ldots$ \\
\hline 214 & 201207080944 & M1.1 & S16W70 & 768 & 784 & $8.7 \mathrm{E}+05$ & {$[10-30]$} & 7.5 & $1.8 \mathrm{E}+31$ & 0.15 & $\ldots$ \\
\hline 215 & 201207081206 & M1.4 & S16W72 & 160 & 1712 & $7.9 \mathrm{E}+05$ & {$[10-30]$} & 5.6 & $3.4 \mathrm{E}+31$ & 0.056 & $\ldots$ \\
\hline 219 & 201207100605 & M2.0 & S16E30 & 1848 & 1456 & $5.0 \mathrm{E}+06$ & [10-30] & 8.1 & $1.2 \mathrm{E}+32$ & 0.038 & 0.15 \\
\hline 222 & 201207171203 & M1.7 & S20W88 & 20740 & 288 & $6.9 \mathrm{E}+06$ & [10-25] & 10.5 & $1.3 \mathrm{E}+31$ & 0.72 & $\ldots$ \\
\hline 223 & 201207190417 & M7.7 & S20W88 & 8532 & 3696 & $3.0 \mathrm{E}+07$ & [10-25] & 5.8 & $2.5 \mathrm{E}+32$ & 0.072 & $\ldots$ \\
\hline 228 & 201208060433 & M1.6 & S14E88 & 728 & 1264 & $1.3 \mathrm{E}+06$ & [10-30] & 5.0 & $9.1 \mathrm{E}+30$ & 0.029 & 0.70 \\
\hline 230 & 201208171312 & M2.4 & N18E88 & 1512 & 2544 & $2.8 \mathrm{E}+06$ & {$[10-30]$} & 5.9 & $5.6 \mathrm{E}+31$ & 0.021 & $\ldots$ \\
\hline 235 & 201208182246 & M1.0 & N18E88 & 1036 & 400 & $7.8 \mathrm{E}+05$ & [10-25] & 8.9 & $1.7 \mathrm{E}+31$ & 0.28 & $\ldots$ \\
\hline 238 & 201209060406 & M1.6 & N04W61 & 2184 & 1456 & $2.0 \mathrm{E}+06$ & {$[10-30]$} & 5.8 & $3.3 \mathrm{E}+31$ & 0.16 & $\ldots$ \\
\hline 241 & 201209300427 & M1.3 & N12W81 & 2228 & 1072 & $2.1 \mathrm{E}+06$ & [10-30] & 5.9 & $3.8 \mathrm{E}+31$ & 0.0073 & $\ldots$ \\
\hline 245 & 201210201805 & M9.0 & S12E88 & 2116 & 12304 & $2.0 \mathrm{E}+07$ & {$[10-30]$} & 6.1 & $8.6 \mathrm{E}+32$ & 0.0089 & $\ldots$ \\
\hline 246 & 201210211946 & M1.3 & S13E78 & 2124 & 976 & $2.7 \mathrm{E}+06$ & {$[10-30]$} & 7.1 & $9.3 \mathrm{E}+31$ & 0.060 & $\ldots$ \\
\hline 248 & 201210230313 & $\mathrm{X} 1.8$ & S13E58 & 1380 & 16543 & $2.9 \mathrm{E}+07$ & [10-25] & 7.0 & $2.5 \mathrm{E}+33$ & 0.0046 & $\ldots$ \\
\hline 251 & 201211122313 & M2.0 & $\mathrm{S} 25 \mathrm{E} 48$ & 2124 & 1840 & $3.2 \mathrm{E}+06$ & {$[10-30]$} & 6.8 & $9.1 \mathrm{E}+31$ & 0.044 & $\ldots$ \\
\hline 253 & 201211130542 & M2.5 & S26E44 & 1396 & 2288 & $3.1 \mathrm{E}+06$ & {$[10-30]$} & 6.6 & $9.1 \mathrm{E}+31$ & 0.072 & 0.83 \\
\hline 255 & 201211140359 & M1.1 & S23E27 & 1352 & 720 & $6.3 \mathrm{E}+05$ & {$[10-30]$} & 3.9 & $2.8 \mathrm{E}+29$ & 6.36 & 0.007 \\
\hline 256 & 201211201236 & M1.7 & N10E22 & 840 & 1200 & $9.7 \mathrm{E}+05$ & {$[10-30]$} & 3.5 & $1.0 \mathrm{E}+30$ & 0.15 & 0.048 \\
\hline 257 & 201211201921 & M1.6 & N10E19 & 372 & 1072 & $5.4 \mathrm{E}+05$ & [10-30] & 4.9 & $2.4 \mathrm{E}+30$ & 1.45 & 0.077 \\
\hline 258 & 201211210645 & M1.4 & N10E12 & 932 & 1136 & $2.0 \mathrm{E}+06$ & {$[10-30]$} & 5.4 & $2.5 \mathrm{E}+31$ & 0.25 & 0.43 \\
\hline 261 & 201211272105 & M1.0 & S13W42 & 1668 & 720 & $9.2 \mathrm{E}+05$ & {$[10-30]$} & 7.3 & $3.0 \mathrm{E}+31$ & 0.075 & 0.71 \\
\hline 262 & 201211282120 & M2.2 & S12W56 & 3044 & 1776 & $4.3 \mathrm{E}+06$ & {$[10-30]$} & 6.6 & $6.8 \mathrm{E}+31$ & 0.18 & $\ldots$ \\
\hline 264 & 201301110843 & M1.2 & N05E42 & 1180 & 880 & $2.0 \mathrm{E}+06$ & [10-25] & 7.0 & $4.8 \mathrm{E}+31$ & 0.066 & 0.24 \\
\hline
\end{tabular}


Table 1

(Continued)

\begin{tabular}{|c|c|c|c|c|c|c|c|c|c|c|c|}
\hline \# & $\begin{array}{c}\text { Flare } \\
\text { Start Time }\end{array}$ & $\begin{array}{c}\text { GOES } \\
\text { Class }\end{array}$ & $\begin{array}{l}\text { Helio- } \\
\text { Graphic } \\
\text { position }\end{array}$ & $\begin{array}{c}\text { Flare } \\
\text { Duration } \\
d \\
(\mathrm{~s})\end{array}$ & $\begin{array}{c}\text { Peak } \\
\text { Counts } \\
P \\
\left(\text { cts s }^{-1}\right)\end{array}$ & $\begin{array}{c}\text { Total } \\
\text { Counts } \\
C \\
\text { (cts) }\end{array}$ & $\begin{array}{c}\text { Fitted } \\
\text { Energy } \\
\text { range } \\
(\mathrm{keV})\end{array}$ & $\begin{array}{c}\text { Cutoff } \\
\text { Energy } \\
e_{\mathrm{wt}} \\
(\mathrm{keV})\end{array}$ & $\begin{array}{c}\text { Nonthermal } \\
\text { Energy } \\
E_{\mathrm{wt}} \\
(\mathrm{erg})\end{array}$ & $\begin{array}{c}\text { Energy } \\
\text { Ratio } \\
E_{\mathrm{th}} / E_{\mathrm{wt}}\end{array}$ & $\begin{array}{c}\text { Energy } \\
\text { Ratio } \\
E_{\mathrm{wt}} / E_{\mathrm{mag}}\end{array}$ \\
\hline 266 & 201301130045 & M1.0 & N18W15 & 764 & 1264 & $6.6 \mathrm{E}+05$ & {$[10-30]$} & 5.4 & $1.1 \mathrm{E}+31$ & $0.17^{*}$ & 0.53 \\
\hline 268 & 201302171545 & M1.9 & N12E23 & 620 & 3312 & $1.5 \mathrm{E}+06$ & {$[10-30]$} & 6.2 & $8.2 \mathrm{E}+30$ & 0.12 & 0.45 \\
\hline 271 & 201303212142 & M1.6 & N09W88 & 3516 & 560 & $3.7 \mathrm{E}+06$ & [10-30] & 4.3 & $1.7 \mathrm{E}+31$ & 0.50 & $\ldots$ \\
\hline 273 & 201304110655 & M6.5 & N11E13 & 1076 & 2160 & $2.8 \mathrm{E}+06$ & {$[10-25]$} & 4.9 & $2.1 \mathrm{E}+31$ & 1.90 & 0.42 \\
\hline 274 & 201304121952 & M3.3 & N21W47 & 2012 & 2928 & $6.5 \mathrm{E}+06$ & [10-30] & 6.5 & $1.5 \mathrm{E}+32$ & 0.094 & $\ldots$ \\
\hline 276 & 201305020458 & M1.1 & N10W19 & 2380 & 448 & $1.3 \mathrm{E}+06$ & [10-30] & 3.1 & $5.6 \mathrm{E}+29$ & 7.36 & 0.009 \\
\hline 277 & 201305031639 & M1.3 & N11W38 & 2872 & 649 & $2.3 \mathrm{E}+05$ & {$[10-30]$} & 4.5 & $2.3 \mathrm{E}+30$ & 0.37 & 0.16 \\
\hline 278 & 201305031724 & M5.7 & N15E83 & 1316 & 3696 & $1.2 \mathrm{E}+07$ & [10-30] & 6.1 & $2.7 \mathrm{E}+32$ & 0.061 & $\ldots$ \\
\hline 283 & 201305122237 & M1.2 & N10E89 & 1872 & 1067 & $4.4 \mathrm{E}+06$ & [10-30] & 7.2 & $2.4 \mathrm{E}+31$ & 0.18 & $\ldots$ \\
\hline 284 & 201305130153 & $\mathrm{X} 1.7$ & N11E89 & 2496 & 13151 & $8.2 \mathrm{E}+07$ & {$[10-30]$} & 6.3 & $6.8 \mathrm{E}+33$ & 0.0033 & $\ldots$ \\
\hline 285 & 201305131157 & M1.3 & N10E89 & 1048 & 1264 & $1.3 \mathrm{E}+06$ & {$[10-30]$} & 6.6 & $7.6 \mathrm{E}+31$ & 0.012 & $\ldots$ \\
\hline 286 & 201305131548 & $\mathrm{X} 2.8$ & N08E89 & 1032 & 33601 & $7.3 \mathrm{E}+07$ & [12-50] & 3.3 & $1.1 \mathrm{E}+31$ & 6.19 & $\ldots$ \\
\hline 288 & 201305150125 & $\mathrm{X} 1.2$ & N10E68 & 3524 & 8656 & $3.9 \mathrm{E}+07$ & [10-25] & 6.4 & $1.5 \mathrm{E}+33$ & 0.026 & $\ldots$ \\
\hline 289 & 201305162136 & M1.3 & N11E40 & 1280 & 624 & $1.5 \mathrm{E}+06$ & [10-30] & 9.8 & $3.7 \mathrm{E}+30$ & 0.96 & 0.17 \\
\hline 291 & 201305200516 & M1.7 & N09E89 & 1380 & 592 & $1.6 \mathrm{E}+06$ & [10-25] & 6.8 & $3.6 \mathrm{E}+31$ & 0.096 & $\ldots$ \\
\hline 292 & 201305221308 & M5.0 & N14W87 & 3248 & 1328 & $1.1 \mathrm{E}+07$ & [10-30] & 4.4 & $1.3 \mathrm{E}+31$ & 1.65 & $\ldots$ \\
\hline 293 & 201305311952 & M1.0 & N12E42 & 1060 & 336 & $5.9 \mathrm{E}+05$ & [10-30] & 6.7 & $4.5 \mathrm{E}+30$ & 1.06 & 2.97 \\
\hline 296 & 201306210230 & M2.9 & S14E73 & 5068 & 912 & $3.7 \mathrm{E}+06$ & [10-25] & 7.4 & $1.2 \mathrm{E}+32$ & 0.12 & $\ldots$ \\
\hline 297 & 201306232048 & M2.9 & S18E63 & 1132 & 2160 & $2.7 \mathrm{E}+06$ & {$[10-30]$} & 5.1 & $3.0 \mathrm{E}+31$ & 0.028 & $\ldots$ \\
\hline 298 & 201307030700 & M1.5 & S14E82 & 1548 & 1008 & $1.9 \mathrm{E}+06$ & [10-30] & 5.1 & $2.1 \mathrm{E}+31$ & 0.26 & $\ldots$ \\
\hline 299 & 201308121021 & M1.5 & S21E17 & 1536 & 976 & $2.2 \mathrm{E}+06$ & [10-25] & 6.5 & $8.5 \mathrm{E}+31$ & 0.071 & 5.07 \\
\hline 303 & 201310110701 & M1.5 & N21E87 & 1124 & 688 & $8.2 \mathrm{E}+05$ & {$[10-30]$} & 4.7 & $5.1 \mathrm{E}+30$ & 0.14 & $\ldots$ \\
\hline 304 & 201310130012 & M1.7 & S22E17 & 1416 & 400 & $8.7 \mathrm{E}+05$ & [8-25] & 5.5 & $2.0 \mathrm{E}+31$ & 0.35 & 0.25 \\
\hline 306 & 201310152331 & M1.3 & S21W22 & 1720 & 912 & $1.0 \mathrm{E}+06$ & [10-30] & 6.8 & $2.0 \mathrm{E}+31$ & 0.19 & 0.52 \\
\hline 307 & 201310171509 & M1.2 & S09W63 & 1696 & 352 & $2.1 \mathrm{E}+06$ & [10-30] & 5.7 & $5.1 \mathrm{E}+30$ & 1.11 & $\ldots$ \\
\hline 308 & 201310220014 & M1.0 & N08E20 & 1068 & 752 & $1.1 \mathrm{E}+06$ & [10-30] & 4.8 & $5.1 \mathrm{E}+31$ & 0.073 & 0.34 \\
\hline 311 & 201310232041 & M2.7 & N08W06 & 3368 & 1904 & $5.4 \mathrm{E}+06$ & [10-30] & 3.2 & $1.9 \mathrm{E}+31$ & 0.33 & 0.11 \\
\hline 312 & 201310232333 & M1.4 & N09W08 & 2000 & 1136 & $1.4 \mathrm{E}+06$ & [10-35] & 5.2 & $1.5 \mathrm{E}+30$ & 2.84 & 0.004 \\
\hline 313 & 201310232358 & M3.1 & N09W09 & 1452 & 2416 & $6.6 \mathrm{E}+06$ & [10-25] & 7.9 & $3.7 \mathrm{E}+31$ & 0.20 & 0.25 \\
\hline 317 & 201310250248 & M2.9 & S07E76 & 3164 & 1840 & $5.5 \mathrm{E}+06$ & [10-30] & 5.8 & $7.0 \mathrm{E}+31$ & 0.16 & $\ldots$ \\
\hline 318 & 201310250753 & $\mathrm{X} 1.7$ & S08E73 & 676 & 10409 & $1.1 \mathrm{E}+07$ & [10-25] & 9.0 & $3.4 \mathrm{E}+33$ & 0.0032 & $\ldots$ \\
\hline 320 & 201310251451 & $\mathrm{X} 2.1$ & S06E69 & 3568 & 16678 & $6.5 \mathrm{E}+07$ & [10-25] & 10.8 & $3.4 \mathrm{E}+32$ & 0.072 & $\ldots$ \\
\hline 321 & 201310251702 & M1.3 & S08E67 & 2052 & 847 & $3.2 \mathrm{E}+06$ & [10-30] & 4.2 & $8.8 \mathrm{E}+30$ & 0.28 & $\ldots$ \\
\hline 324 & 201310260559 & M2.3 & S08E59 & 1880 & 2032 & $3.4 \mathrm{E}+06$ & [10-20] & 4.9 & $1.4 \mathrm{E}+31$ & 0.24 & $\ldots$ \\
\hline 325 & 201310260917 & M1.5 & S08E59 & 1060 & 320 & $6.5 \mathrm{E}+05$ & [10-30] & 5.4 & $4.3 \mathrm{E}+30$ & 0.67 & $\ldots$ \\
\hline 326 & 201310261048 & M1.8 & S06E59 & 1176 & 320 & $1.0 \mathrm{E}+06$ & [10-30] & 7.3 & $5.5 \mathrm{E}+31$ & 0.14 & $\ldots$ \\
\hline 328 & 201310261949 & M1.0 & S08E51 & 1940 & 272 & $6.2 \mathrm{E}+05$ & [10-25] & 6.6 & $1.4 \mathrm{E}+30$ & 0.60 & $\ldots$ \\
\hline 330 & 201310280141 & $\mathrm{X} 1.0$ & N05W72 & 2376 & 9863 & $3.1 \mathrm{E}+07$ & [10-20] & 6.9 & $1.9 \mathrm{E}+32$ & 0.12 & $\ldots$ \\
\hline 332 & 201310281132 & M1.4 & S14W46 & 3956 & 309 & $2.3 \mathrm{E}+06$ & [10-30] & 8.6 & $5.0 \mathrm{E}+31$ & 0.11 & $\ldots$ \\
\hline 334 & 201310281446 & M2.7 & S08E27 & 2600 & 2288 & $8.8 \mathrm{E}+06$ & [10-30] & 6.5 & $1.3 \mathrm{E}+32$ & 0.24 & 2.53 \\
\hline 336 & 201310282048 & M1.5 & N07W83 & 1748 & 1200 & $1.5 \mathrm{E}+06$ & [10-30] & 7.0 & $4.9 \mathrm{E}+31$ & 0.037 & $\ldots$ \\
\hline 340 & 201311022213 & M1.6 & S12W12 & 768 & 1200 & $1.6 \mathrm{E}+06$ & [10-30] & 6.4 & $6.4 \mathrm{E}+31$ & 0.037 & 0.73 \\
\hline 343 & 201311051808 & M1.0 & S12E47 & 1124 & 688 & $8.2 \mathrm{E}+05$ & [10-30] & 6.1 & $1.2 \mathrm{E}+31$ & 0.12 & $\ldots$ \\
\hline 345 & 201311061339 & M3.8 & S09E35 & 1936 & 2928 & $6.0 \mathrm{E}+06$ & [10-30] & 6.0 & $1.1 \mathrm{E}+32$ & 0.043 & 0.64 \\
\hline 347 & 201311070334 & M2.3 & S08E26 & 1436 & 1776 & $1.7 \mathrm{E}+06$ & [10-25] & 5.1 & $4.8 \mathrm{E}+31$ & 0.15 & 0.13 \\
\hline 351 & 201311100508 & $\mathrm{X} 1.1$ & S11W17 & 3284 & 9303 & $1.3 \mathrm{E}+07$ & [10-30] & 8.0 & $1.3 \mathrm{E}+33$ & 0.043 & 4.95 \\
\hline 352 & 201311111101 & M2.4 & S17E74 & 3068 & 1264 & $6.6 \mathrm{E}+06$ & [10-30] & 7.3 & $2.3 \mathrm{E}+32$ & 0.032 & $\ldots$ \\
\hline 353 & 201311131457 & M1.4 & S20E46 & 1400 & 592 & $1.3 \mathrm{E}+06$ & [10-30] & 8.1 & $4.1 \mathrm{E}+31$ & 0.25 & $\ldots$ \\
\hline 354 & 201311150220 & M1.0 & N07E53 & 1252 & 656 & $9.0 \mathrm{E}+05$ & [10-30] & 6.9 & $4.5 \mathrm{E}+31$ & 0.086 & $\ldots$ \\
\hline 357 & 201311170506 & M1.0 & S19W41 & 1208 & 592 & $5.4 \mathrm{E}+05$ & [10-25] & 7.0 & $2.4 \mathrm{E}+31$ & 0.025 & 0.20 \\
\hline 359 & 201311211052 & M1.2 & S14W89 & 1248 & 448 & $2.1 \mathrm{E}+06$ & [10-25] & 5.7 & $3.5 \mathrm{E}+31$ & 0.040 & $\ldots$ \\
\hline 360 & 201311230220 & M1.1 & N13W58 & 2584 & 432 & $1.6 \mathrm{E}+06$ & [10-30] & 4.1 & $8.2 \mathrm{E}+31$ & 0.034 & $\ldots$ \\
\hline 363 & 201312192306 & M3.5 & S16E89 & 2304 & 2160 & $5.5 \mathrm{E}+06$ & [10-30] & 7.0 & $2.8 \mathrm{E}+32$ & 0.055 & $\ldots$ \\
\hline 364 & 201312201135 & M1.6 & S16E78 & 4272 & 336 & $2.1 \mathrm{E}+06$ & {$[10-30]$} & 5.3 & $9.6 \mathrm{E}+30$ & 0.37 & $\ldots$ \\
\hline 365 & 201312220805 & M1.9 & S17W51 & 1788 & 1776 & $2.2 \mathrm{E}+06$ & [10-30] & 8.2 & $4.2 \mathrm{E}+31$ & 0.054 & $\ldots$ \\
\hline 366 & 201312220833 & M1.1 & S17W52 & 1956 & 320 & $5.9 \mathrm{E}+05$ & [10-25] & 5.8 & $8.4 \mathrm{E}+30$ & 0.32 & $\ldots$ \\
\hline 367 & 201312221424 & M1.6 & S16E44 & 2532 & 416 & $1.7 \mathrm{E}+06$ & [10-30] & 5.3 & $5.1 \mathrm{E}+31$ & 0.14 & 0.79 \\
\hline 368 & 201312221506 & M3.3 & S17W55 & 1328 & 1968 & $3.4 \mathrm{E}+06$ & [10-30] & 6.4 & $3.0 \mathrm{E}+31$ & 0.37 & $\ldots$ \\
\hline 377 & 201401031241 & M1.0 & S04E52 & 1000 & 30 & $9.5 \mathrm{E}+04$ & [10-30] & 3.6 & $2.0 \mathrm{E}+29$ & 5.44 & $\ldots$ \\
\hline 379 & 201401041016 & M1.3 & S05E49 & 2888 & 400 & $2.0 \mathrm{E}+06$ & [10-30] & 5.4 & $2.0 \mathrm{E}+31$ & 0.23 & $\ldots$ \\
\hline 382 & 201401070349 & M1.0 & N07E07 & 1432 & 880 & $8.1 \mathrm{E}+05$ & [10-25] & 7.3 & $2.9 \mathrm{E}+31$ & 0.051 & 0.39 \\
\hline
\end{tabular}


Table 1

(Continued)

\begin{tabular}{|c|c|c|c|c|c|c|c|c|c|c|c|}
\hline \# & $\begin{array}{c}\text { Flare } \\
\text { Start Time }\end{array}$ & $\begin{array}{l}\text { GOES } \\
\text { Class }\end{array}$ & $\begin{array}{l}\text { Helio- } \\
\text { Graphic } \\
\text { position }\end{array}$ & $\begin{array}{c}\text { Flare } \\
\text { Duration } \\
d \\
(\mathrm{~s})\end{array}$ & $\begin{array}{c}\text { Peak } \\
\text { Counts } \\
P \\
\left(\text { cts s }^{-1}\right)\end{array}$ & $\begin{array}{c}\text { Total } \\
\text { Counts } \\
C \\
\text { (cts) }\end{array}$ & $\begin{array}{l}\text { Fitted } \\
\text { Energy } \\
\text { range } \\
(\mathrm{keV})\end{array}$ & $\begin{array}{c}\text { Cutoff } \\
\text { Energy } \\
e_{\mathrm{wt}} \\
(\mathrm{keV})\end{array}$ & $\begin{array}{c}\text { Nonthermal } \\
\text { Energy } \\
E_{\mathrm{wt}} \\
(\mathrm{erg})\end{array}$ & $\begin{array}{c}\text { Energy } \\
\text { Ratio } \\
E_{\mathrm{th}} / E_{\mathrm{wt}}\end{array}$ & $\begin{array}{c}\text { Energy } \\
\text { Ratio } \\
E_{\mathrm{wt}} / E_{\mathrm{mag}}\end{array}$ \\
\hline 383 & 201401071007 & M7.2 & S13E13 & 2000 & 7967 & $2.8 \mathrm{E}+07$ & [16-30] & 8.7 & $2.4 \mathrm{E}+33$ & 0.0076 & 4.47 \\
\hline 385 & 201401080339 & M3.6 & N11W88 & 2016 & 2672 & $4.4 \mathrm{E}+06$ & [10-30] & 5.4 & $1.5 \mathrm{E}+32$ & 0.0057 & $\ldots$ \\
\hline 386 & 201401132148 & M1.3 & S08W75 & 660 & 1456 & $7.8 \mathrm{E}+05$ & [10-25] & 8.3 & $3.4 \mathrm{E}+31$ & 0.013 & $\cdots$ \\
\hline 387 & 201401270105 & M1.0 & S16E88 & 2860 & 272 & $1.5 \mathrm{E}+06$ & [10-30] & 3.3 & $4.5 \mathrm{E}+29$ & 10.74 & $\cdots$ \\
\hline 389 & 201401272205 & M4.9 & S14E88 & 1880 & 4129 & $5.8 \mathrm{E}+06$ & {$[10-30]$} & 9.8 & $2.6 \mathrm{E}+32$ & 0.0064 & $\ldots$ \\
\hline 393 & 201401281233 & M1.3 & S15E79 & 1708 & 560 & $1.2 \mathrm{E}+06$ & {$[10-30]$} & 9.1 & $1.7 \mathrm{E}+31$ & 0.020 & $\ldots$ \\
\hline 395 & 201401282204 & M2.6 & S14E74 & 1112 & 1968 & $2.5 \mathrm{E}+06$ & [10-30] & 6.5 & $1.3 \mathrm{E}+32$ & 0.0055 & $\cdots$ \\
\hline
\end{tabular}

Note. The soft X-ray flare duration $d$ (Column 5), the peak counts $P$ (Column 6), the total counts $C$ (Column 7), the fitted energy range (Column 8), the (warm-target) lower cutoff energy $e_{w t}$ for a mean temperature of $T_{e}=8.6 \mathrm{MK}$ in flaring active regions (Column 9), the (warm-target) nonthermal energy $E_{\mathrm{wt}}($ Column 10$)$, the ratio of the thermal energy $E_{\mathrm{th}}$ to the (warm-target) nonthermal energy $E_{\mathrm{wt}}$ (Column 11), and the ratio of the (warm-target) nonthermal energy $E_{\mathrm{wt}}$ to the magnetic energy $E_{\text {mag }}$ (Column 12). Questionable solar flare events, detected in the front detectors without position, are flagged with a (*) sign (in Column 11)

(This table is available in machine-readable form.)

\subsection{Thermal-Nonthermal Cross-over Energy}

The bremsstrahlung spectrum $I(\varepsilon)$ of a thermal plasma with temperature $T$, as a function of the photon energy $\varepsilon=h \nu$, setting the coronal electron density equal to the ion density ( $n=n_{i}=n_{e}$ ), and neglecting factors of the order of unity (such as the Gaunt factor $g(\nu, T)$ in the approximation of the Bethe-Heitler bremsstrahlung cross-section), and the ion charge number, $Z \approx 1$, is (Brown 1974; Dulk \& Dennis 1982),

$$
I(\varepsilon)=I_{0} \int \frac{\exp \left(-\varepsilon / k_{B} T\right)}{T^{1 / 2}} \frac{d E M(T)}{d T} d T,
$$

where $I_{0} \approx 8.1 \times 10^{-39} \mathrm{keV} \mathrm{s}^{-1} \mathrm{~cm}^{-2} \mathrm{keV}^{-1}$ and $d E M(T) / d T$ specifies the differential emission measure (DEM) $n^{2} d V$ in the element of volume $d V$ corresponding to a temperature range of $d T$,

$$
\left(\frac{d E M(T)}{d T}\right) d T=n^{2}(T) d V .
$$

Regardless, whether we define this DEM distribution by an isothermal or by a multithermal plasma (Aschwanden 2007), the thermal spectrum $I(\varepsilon)$ falls off similarly to an exponential function at an energy of $\varepsilon \lesssim 20 \mathrm{keV}$ (or up to $\lesssim 40 \mathrm{keV}$ in extremal cases), while the nonthermal spectrum in the higher energy range of $\varepsilon \approx 20-100 \mathrm{keV}$ can be approximated with a single (or broken) power-law function (Equation (1)).

Because of the two different functional shapes, a cross-over energy $\varepsilon_{c}$ can often be defined from the change in the spectral slope between the thermal and the nonthermal spectral component. The electron energy spectrum, however, can have a substantially lower or higher cutoff energy (e.g., Holman 2003).

We represent the combined spectrum with the sum of the (exponential-like) thermal and the (power-law-like) nonthermal component, i.e.,

$$
\begin{aligned}
I(\varepsilon) & =I_{\mathrm{th}}(\varepsilon)+I_{\mathrm{nt}}(\varepsilon) \\
& =I_{0} \int \frac{\exp \left(-\varepsilon / k_{B} T\right)}{T^{1 / 2}} \frac{d E M(T)}{d T} d T+A \varepsilon^{-\gamma},
\end{aligned}
$$

where the cross-over energy $\varepsilon_{\mathrm{co}}$ can be determined in the (bestfit) model spectrum $I(\varepsilon)$ from the energy where the logarithmic slope is steepest, i.e., from the maximum of $\partial \log I(\varepsilon) / \partial \log \varepsilon$.

\subsection{Warm-target Model}

A new theoretical model has recently been developed that allows us to calculate the low-energy cutoff energy in the thicktarget model directly, by including the "warming" of the cold thick-target plasma during the electron precipitation phase, when chromospheric heating and evaporation sets in (Kontar et al. 2015). Previous applications of the thick-target model generally assume cold (chromospheric) temperatures in the electron precipitation site (e.g., Holman et al. 2011, for a review). The theoretical derivation of the warm-target model has been analytically derived and tested with numerical simulations that include the effects of collisional energy diffusion and thermalization of fast electrons (Galloway et al. 2005; Goncharov et al. 2010; Jeffrey et al. 2014). According to this model, the effective low-energy cutoff $e_{c}$ is a function of the temperature $e_{\mathrm{th}}=k_{B} T_{e}$ of the warm-target plasma and the power-law slope $\delta=\gamma+1$ of the (nonthermal) electron flux,

$$
e_{c} \approx(\xi+2) k_{B} T_{e}=\delta k_{B} T_{e} .
$$

where $\xi=\gamma-1$ is the power-law slope of the sourceintegrated mean electron flux spectrum (see Equations (8)(10) in Kontar et al. 2015), and $T_{e}$ is the temperature of the warm target, which is a mixture or the cold preflare plasma and the heated evaporating plasma. Thus, for the temperature range of a medium-sized to a large X-class flare, which spans $T_{e} \approx 10-25 \mathrm{MK}$, the temperature in energy units is $E_{\mathrm{th}}=k_{B} T_{e} \approx 0.9-2.1 \mathrm{keV}$, and for a range of power-law slopes of $\delta=3-6$ (Dennis 1985; Kontar et al. 2011), a range of $e_{c} \approx 3-13 \mathrm{keV}$ is predicted for the low-energy cutoffs by this model.

Besides collisional heating of the warm chromospheric target, electron beams and beam-driven Langmuir wave turbulence may affect the low-energy cutoff additionally (Hannah et al. 2009). Alternative analytical models on the 
low-energy cutoff can be derived from a collisional time-offlight model (Appendix A), from the Rosner-Tucker-Vaiana heating/cooling balance model (Appendix B), and from the runaway acceleration model (Appendix $\mathrm{C}$ ).

\section{DATA ANALYSIS METHOD}

From the same comprehensive catalog of $399 \mathrm{M}$ and X-class flares observed with $S D O$ during 2010-2014, used in the first two studies of our global flare energetics project, we will analyze all events that have been simultaneously observed in hard X-rays and gamma rays with RHESSI. The orbit of RHESSI has a duty cycle of $\approx 50 \%$, leading to a total of 191 events that have suitable time coverage. In the following, we describe the analysis of these events, which are also listed in Table 1 (labeled with identical identification numbers \#1-399 as used in Papers I and II). We explain the various steps performed in our analysis for three examples shown in Figures 1-3.

\subsection{Spectral Modeling of RHESSI Data with OSPEX}

For the measurement of the nonthermal energy $\left(E_{\mathrm{nt}}\right)$ of electrons during solar flares, we use the OSPEX (Object Spectral Executive) software, which is an object-oriented interface for X-ray spectral analysis of solar data, written by Richard Schwartz and others (see the RHESSI website at http://hesperia.gsfc.nasa.gov/ for documentation). The OSPEX software allows the user to read RHESSI data, to select and subtract a background, to select time intervals of interest, to select a combination of photon flux model components, and to fit those components to the spectrum in each selected time interval. During the fitting process, the response matrix is used to convert the photon model to the model counts that are fitted to the observed counts. The OSPEX software deals also with changes of attenuator states, decimation, pulse pile-up effects, and albedo effects, and provides procedures to calculate the nonthermal energy $\left(E_{\mathrm{nt}}\right)$ (according to the thick-target model) and the thermal energy $\left(E_{\mathrm{th}}\right)$ down to energies of $\gtrsim 3 \mathrm{keV}$.

RHESSI complements spectral information of the DEM distribution at the high-temperature side $\left(T_{e} \gtrsim 16 \mathrm{MK}\right)$ (Caspi 2010; Caspi \& Lin 2010; Caspi et al. 2014), while AIA/SDO provides DEM information at the low-temperature side $\left(T_{e} \lesssim 16 \mathrm{MK}\right)$, as we determined in Paper II. For spectral modeling, we are using the two-component model vth thick2_vnorm, which includes a thermal component at low energies and a (broken) power-law function at higher (nonthermal) energies. In our spectral fits, we are only interested in the transition from the thermal to the nonthermal spectrum, which can be expressed by an exponential-like plus a single-power-law function (Equation (7)), and thus we use only the lower power-law part of the two-component model vth +thick2_vnorm, while the spectral slope in the upper part was set to a constant $\left(\delta_{2}=4\right)$. In addition, we use calc_nontherm_electron_energy_flux of the OSPEX package to calculate the nonthermal energy flux in the thick-target model.

RHESSI Spectral Fitting Range Selection: in order to obtain a self-consistent measure of the nonthermal energy, which varies considerably during the duration of a flare or among different flares, we have to choose a spectral fitting range that covers a sufficient part of both the thermal and nonthermal components. We choose the maximum energy range $\left[\varepsilon_{1}, \varepsilon_{2}\right]$, bound by $\varepsilon_{1}=6 \ldots 10 \mathrm{keV}$ and $\varepsilon_{2}=20 \ldots 50 \mathrm{keV}$, in which an acceptable (reduced) $\chi^{2}$-value $(\chi<2.0)$ is obtained for the spectral fit. The upper bound of the fitting range is mostly constrained by the photon count statistics, which is often too noisy for energies at $\varepsilon_{2} \gtrsim 30 \mathrm{keV}$ during small flares (M-class here), given the time steps of $\Delta t=20 \mathrm{~s}$ chosen throughout. The fitted energy ranges also cover the range of cross-over energies (10-28 keV) found in multithermal fitting of energydependent time delays (Aschwanden 2007).

As a general criticism, we have to be aware that the nonthermal spectral component could in addition also be confused with a multithermal component in the fitted spectral range of $\varepsilon \approx 10-30 \mathrm{keV}$ (Aschwanden 2007), or with nonuniform ionization effects ( $\mathrm{Su}$ et al. 2011), or with returncurrent losses (Holman 2012).

RHESSI Detector Selection: we used the standard option of OSPEX, where a spectral fit is calculated from the combined counts of a selectable set of RHESSI subcollimaters. RHESSI has nine (subcollimator) detectors that originially had nearidentical sensitivities, but progressively deviated from each other as a result of steady degradation over time due to radiation damage from charged particles. Heating up the germanium restores the lost sensitivity and resolution, and thus five annealing procedures have been applied to RHESSI so far (second anneal at 2010 March 16-May 1; third at 2012 January 17-February 22; forth at 2014 June 26-August 13; and fifth at 2016 February 23-April 23). No science data are collected during the annealing periods. Based on the performance of the individual detector sensitivities, it is general practice to exclude detectors 2 and 7 in spectral fits. Furthermore, detectors 4 and 5 are considered to be unreliable after 2012 January (R. Schwartz 2016, private communication). Therefore, we select the set of detectors $[1,3,4,5,6,8,9]$ in spectral fits up to the third anneal in 2012 January (events \# 1-126 in Table 1), and the set of $[1,3,6,8,9]$ after 2012 February (events \# 154-395 in Table 1). Omitting detectors 4 and 5 in the latter set of 71 events yields a total nonthermal energy that is by a factor of $q_{\mathrm{det}}=E_{\mathrm{nt}}[1,3,6,8,9] / E_{\mathrm{nt}}[1,3,4,5,6,8,9]=1.3 \pm 0.5$ higher.

GOES Time Range and RHESSI Time Resolution: we download the GOES 1-8 $\AA$ light curves $F_{G O E S}(t)$ and calculate the time derivative as a proxy for the hard $\mathrm{X}$-ray time profile $F_{\mathrm{HXR}} \approx d F_{G O E S} / d t$, as shown in Figures 1(a), 2(a), and 3(a). The start time $t_{\text {start }}$, peak time $t_{\text {peak }}$, and end time $t_{\text {end }}$ are defined from the NOAA/GOES catalog. We compute consecutive spectra in time steps of $\Delta t=20 \mathrm{~s}$. Note that RHESSI is a spinning spacecraft with a period of $4 \mathrm{~s}$, which does not cause any modulation effects for $20 \mathrm{~s}$ time integrations.

RHESSI Quick-look Data: in a next step, we inspect the RHESSI quick-look time profiles (Figures 1(b), 2(b), and 3(b)), which show photon counts in different energy channels in the range of $6-300 \mathrm{keV}$. Based on these RHESSI time profiles, we select time intervals for background subtraction. Generally, we select a time interval at flare start as the background interval (in $90 \%$ ), and subtract this preflare spectrum for the entire flare time interval. Only in a few cases $(10 \%)$ where the preflare flux is higher than the postflare flux, we choose a time interval at flare end for background subtraction. The RHESSI quick-look data show changes in the attenuator state (e.g., Figures 2(b), 3(b)), which are automatically handled in most time intervals 

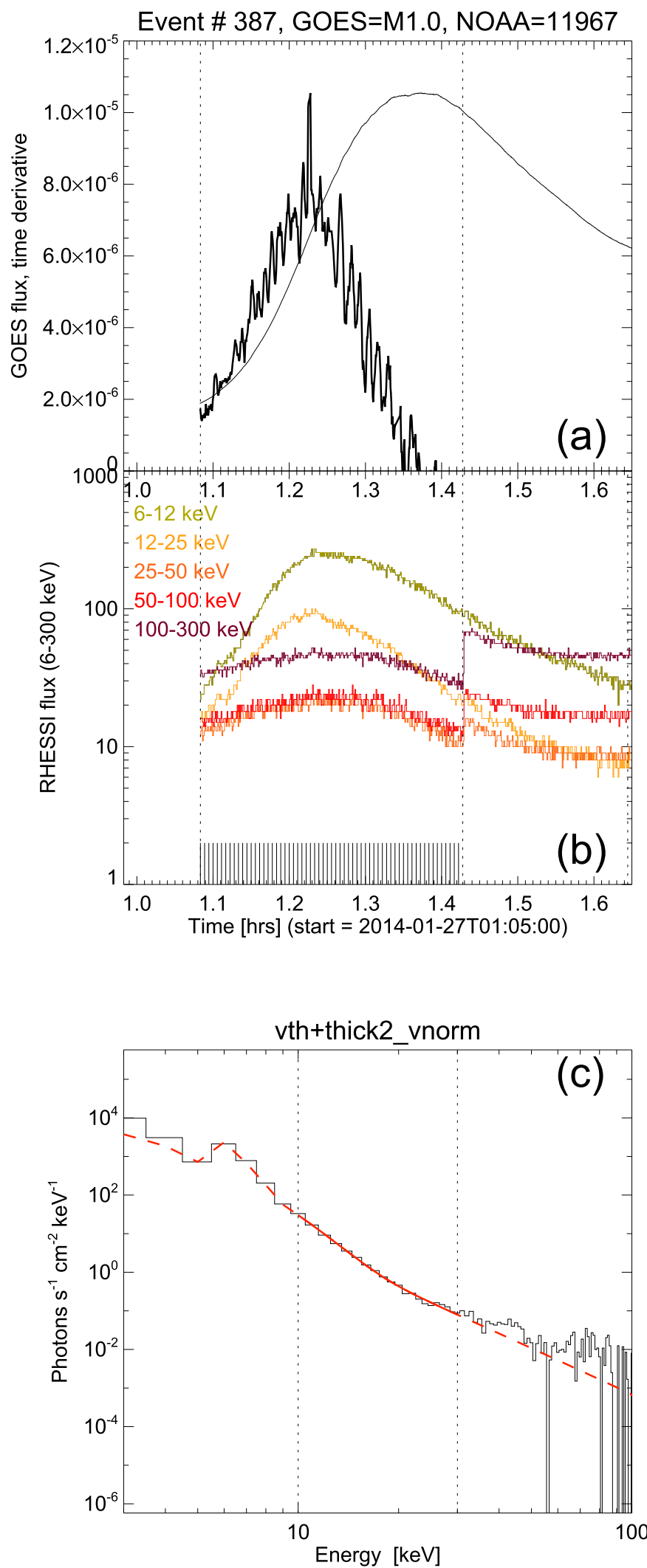
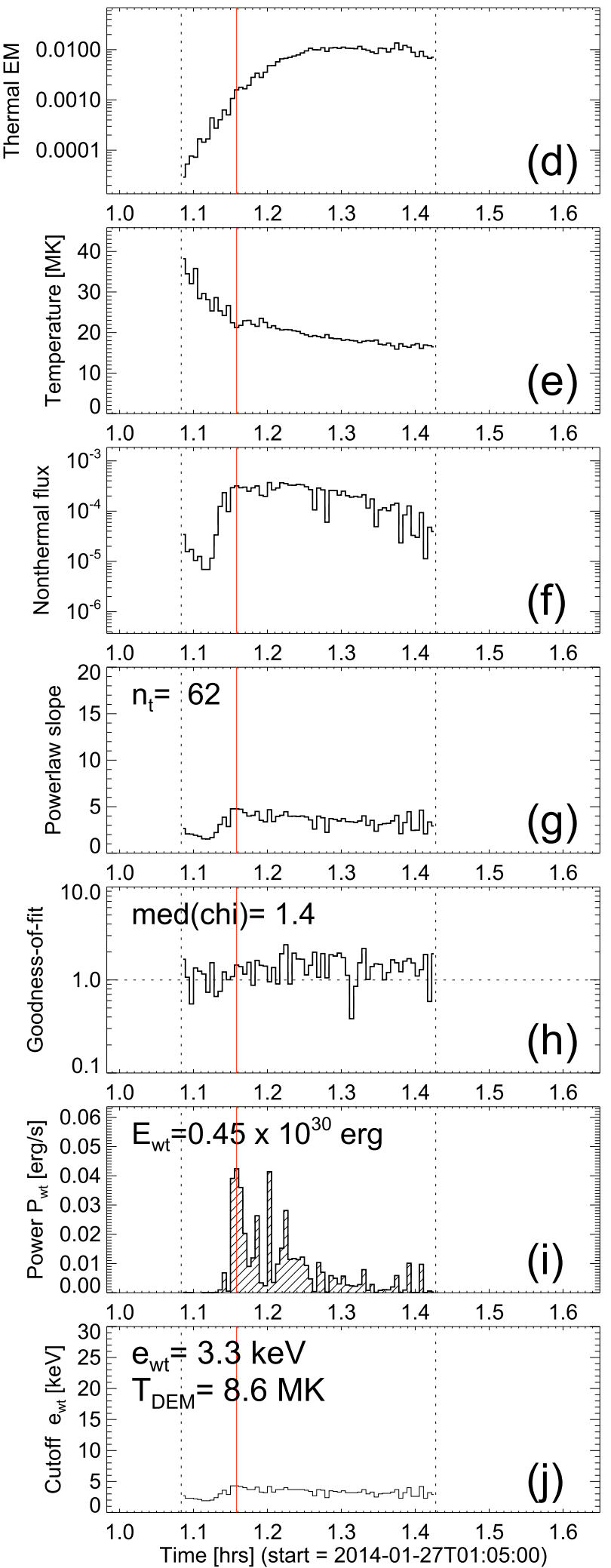

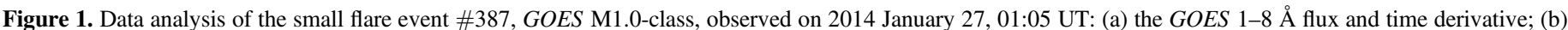

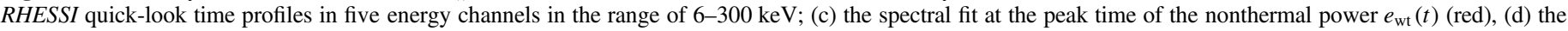

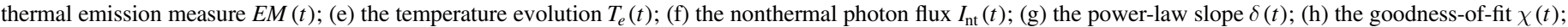

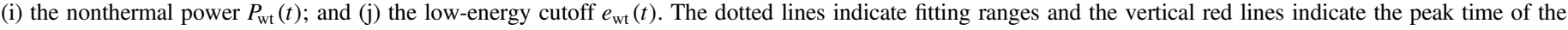
nonthermal power. 

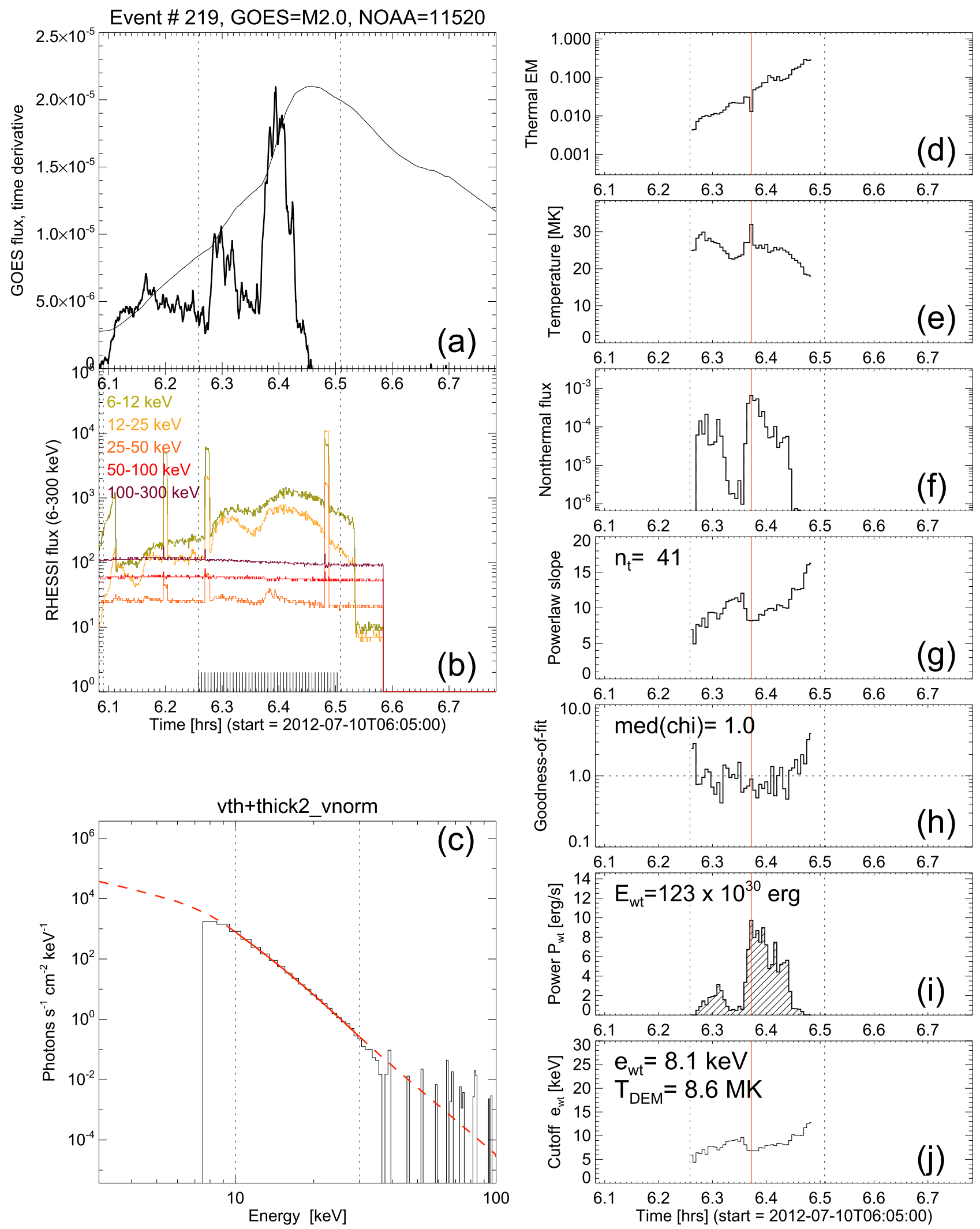

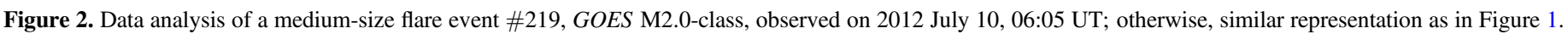

with the OSPEX software, unless there is a change in the attenuator state during a selected time interval itself, in which case this time interval is removed from the spectral analysis.
The quick-look data occasionally show data gaps that are caused when RHESSI enters spacecraft night in its near-Earth orbit. If the data gap does not occur during the flare peak of 

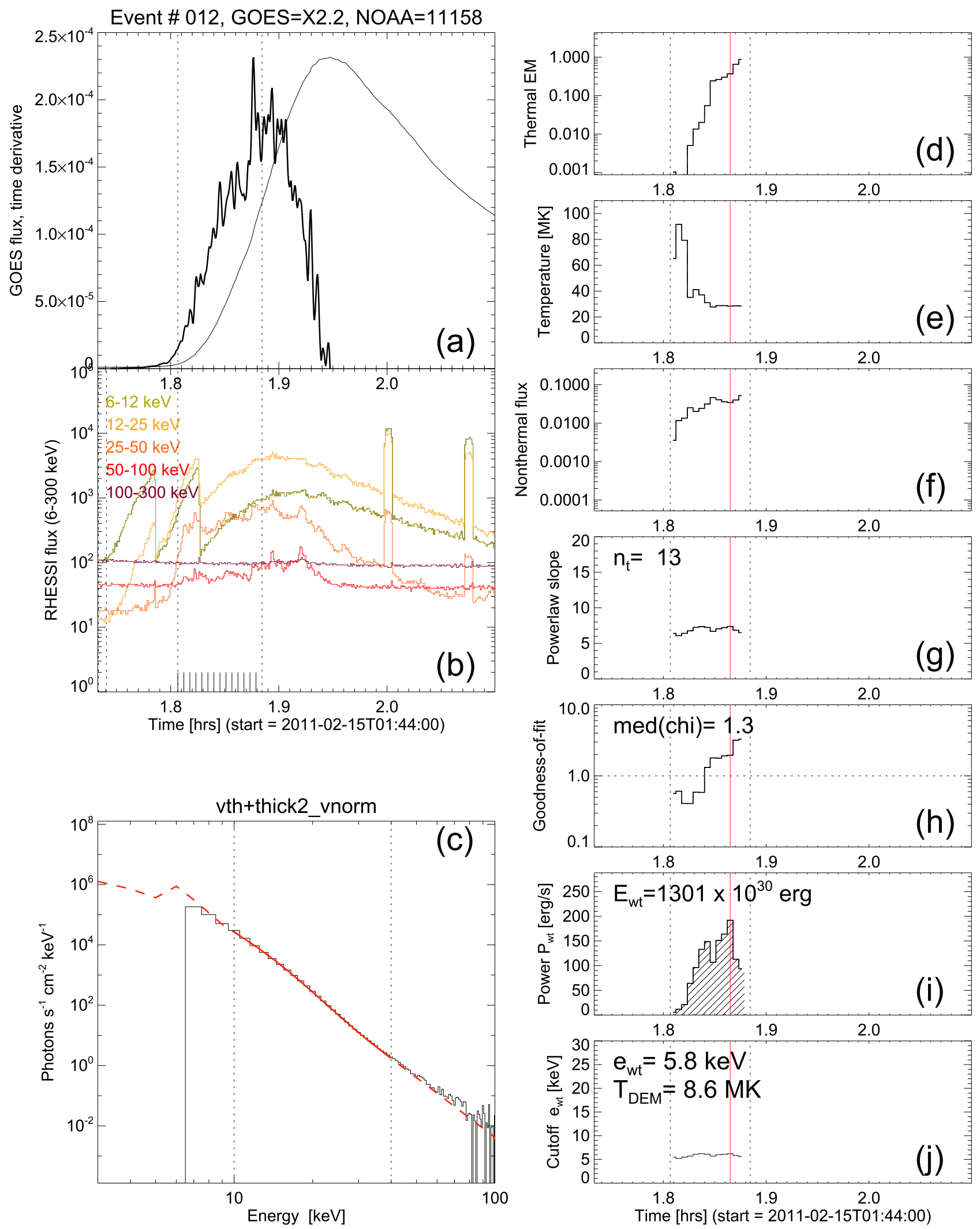

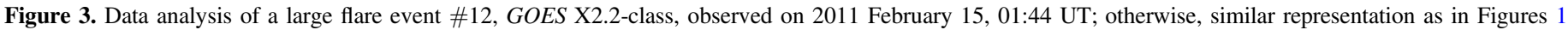
and 2 .

hard X-ray emission, we still include the event in the analysis, as long as the time interval of dominant nonthermal HXR emission is covered (such as in event \#219 in Figure 2(b)).
OSPEX Spectral Fitting: for spectral fitting, we perform first a semi-calibration and store the detector response matrix (DRM), and then run a spectral fit with the fit function vth 

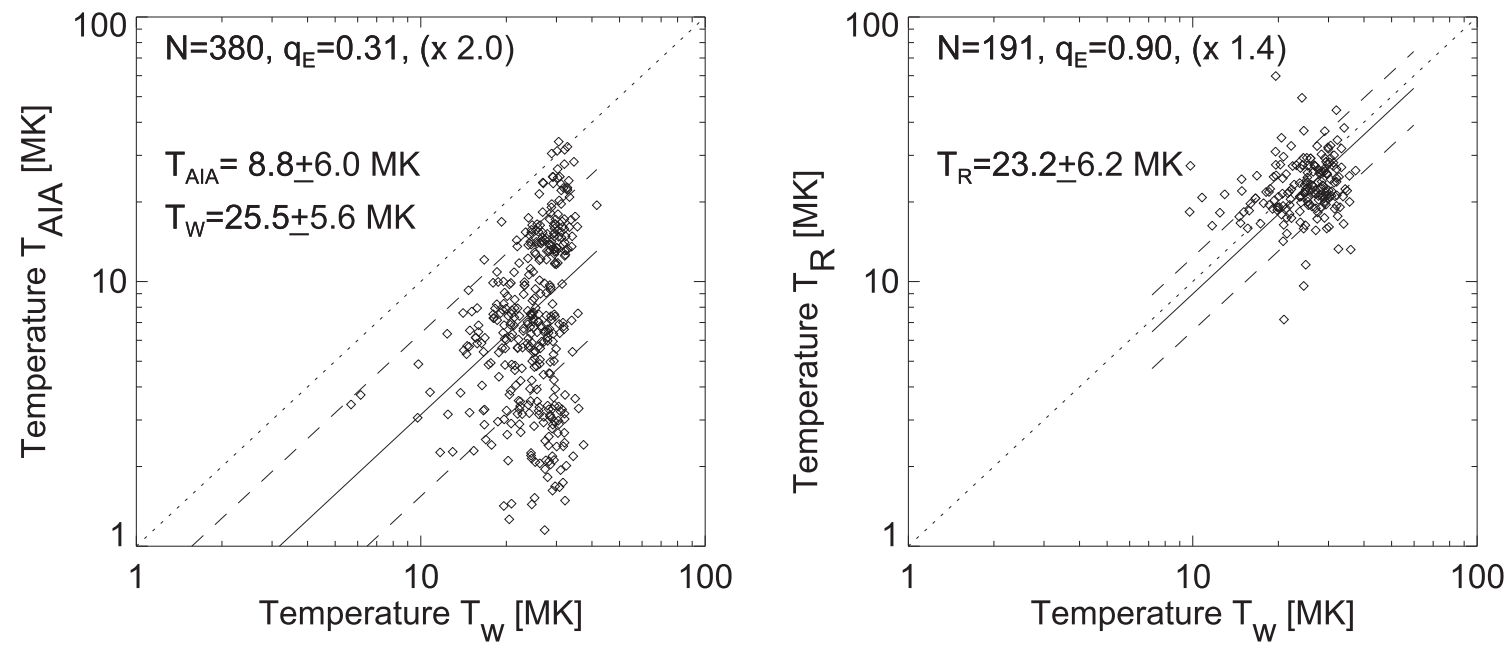

Figure 4. Comparison of three different temperature definitions: the DEM peak temperature $T_{\mathrm{AIA}}$ as a function of the DEM-weighted temperature $T_{w}$ as measured in Paper II (left-hand panel), and the time-averaged RHESSI temperature $T_{R}$ as a function of $T_{w}$ (right-hand panel). The (logarithmically) averaged temperature ratio is indicated with a solid line, the logarithmic standard deviation is indicated with two dashed lines, and the unity ratio is indicated with a dotted line.
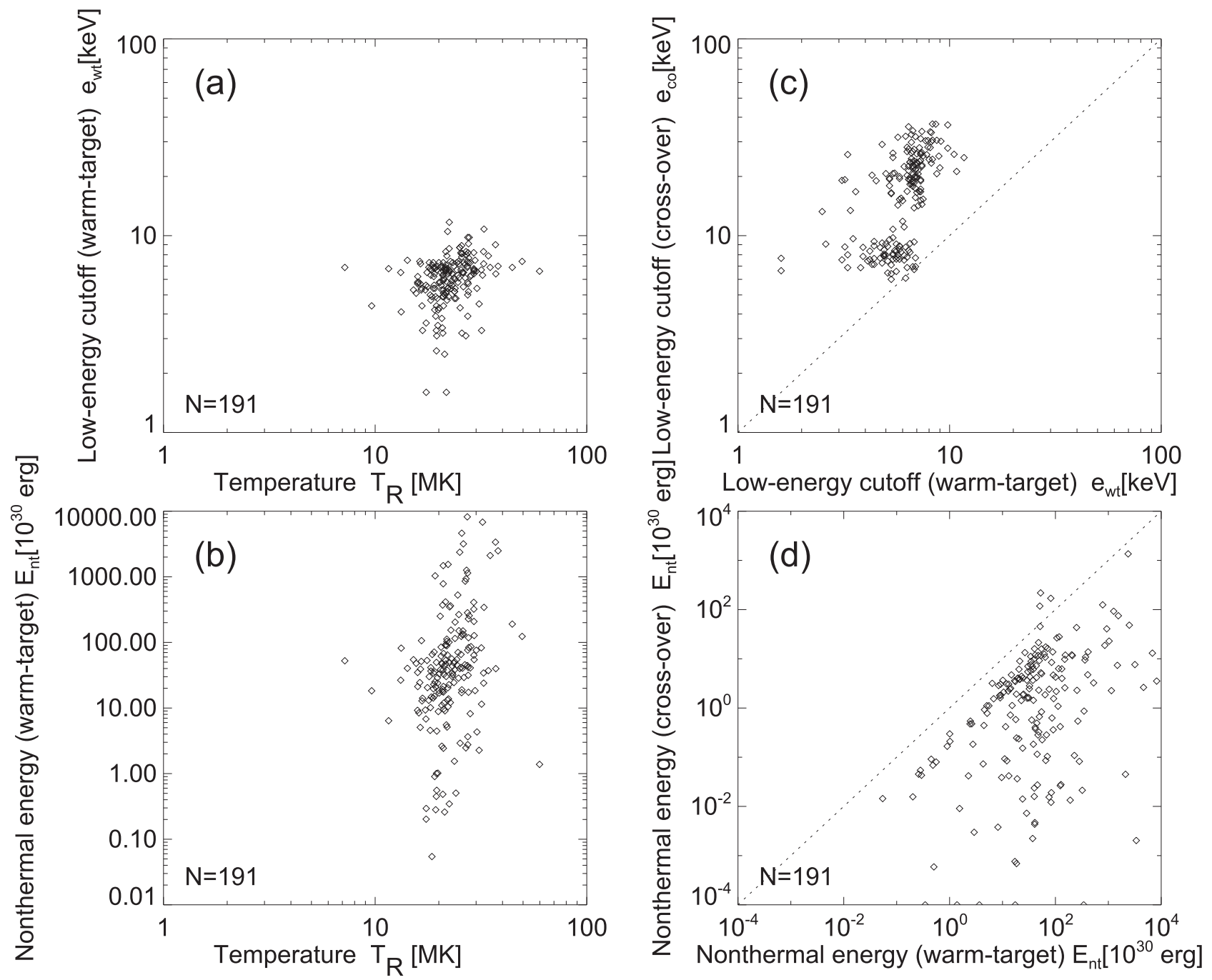

Figure 5. Functional dependence of the low-energy cutoff $e_{\mathrm{wt}}(\mathrm{a})$, and the (warm-target) nonthermal energy $E_{\mathrm{nt}}(\mathrm{b})$ as a function of the RHESSI temperature $T_{R}$. Scatter plots of the low-energy cutoffs (c) and the nonthermal energies (d) are shown between the warm-target and the cross-over model. The diagonal dotted lines (in righthand panels) indicate equivalence. Note that the cross-over method yields systematically larger cutuoff energies and smaller nonthermal energies than the warm-target model. 
+thick2_vnorm using the OSPEX software, optimizing the following model fit parameters (for each time interval $t$ ):

\begin{tabular}{ll}
\hline \hline$E M(t)$ & $=$ emission measure in units of $10^{49} \mathrm{~cm}^{-3}$ \\
$T_{e}(t)$ & $=$ plasma temperature in units of $\mathrm{keV}(1 \mathrm{keV}=11.6 \mathrm{MK})$ \\
$A(t)$ & $=$ photon flux at $\varepsilon=50 \mathrm{keV}$ \\
$\delta(t)$ & $=$ negative power-law index of electron spectrum \\
$e_{c}(t)$ & $=$ low-energy cutoff
\end{tabular}

Examples of spectral fits are shown in Figures 1(c), 2(c), and 3(c), fitted at the time of the peak power $P_{\mathrm{co}}(t)$ (indicated with red vertical lines in Figures 1, 2, and 3). The best-fit spectrum yields a cross-over energy $e_{\mathrm{co}}$ between the thermal and nonthermal spectral component. Alternatively, the warm-target model of Kontar et al. (2015) yields a low-energy cutoff value $e_{\mathrm{wt}}$. The fitted energy ranges are listed in Table 1 and are indicated with dotted vertical lines in Figures $1(\mathrm{~g}), 2(\mathrm{~g})$, and $3(\mathrm{~g})$. The goodness-of-fit is quantified with the $\chi^{2}$-value criterion. In the case of bad fits of the $\chi^{2}$-values $(\chi>2)$, we changed either the fitted energy range (in 13\%), the selected interval for background subtraction $(10 \%)$, or the fitted time range $(5 \%)$.

\section{RESULTS}

The numerical values of the main results of the low-energy cutoffs $e_{c}$ (which we label as $e_{\text {co }}$ in the cross-over method, and as $e_{\mathrm{wt}}$ in the warm-target method), and the nonthermal energy $E_{\mathrm{nt}}$ for the analyzed 191 events are listed in Table 1, while scatter plots and distributions are shown in Figures 4-8.

\subsection{Time Evolution of Flares}

Three examples of analyzed flare events are shown in Figures 1, 2, and 3, including one of the smallest events (Figure 1: \#387, GOES M1.0 class), an event with multi-peak characteristics (Figure 2; \#219, GOES M2.0 class), and one of the largest events (Figure 3; \#12, GOES X2.2 class). In all three cases, we show the time evolution of the most important fit parameters in the various panels ((d) through (j)) of Figures 1-3: (d) the thermal emission measure $E M(t)$; (e) the temperature evolution $T_{e}(t)$; (f) the nonthermal photon flux $I_{\text {nt }}(t)$ at $50 \mathrm{keV}$; $(\mathrm{g})$ the power-law slope $\delta(t)$; (h) the goodnessof-fit $\chi(t)$; (i) the nonthermal power $P_{\mathrm{wt}}(t)$ using the low cutoff energy based on the warm-target model (Section 2.3); and (j) the low-energy cutoff $e_{\mathrm{wt}}(t)$ of the warm-target model. In the examples shown in Figures 1, 2 and 3, we see that the thermal emission measure $E M(t)$ increases during the rise time of the GOES flux, while the temperature $T_{e}(t)$ decreases, which indicates both, namely density and temperature increases due to chromospheric evaporation, as well as subsequent plasma cooling, during the impulsive flare phase. Since multiple heating and cooling cycles overlap during a flare, we see both effects simultaneously. The cases shown in Figures 1, 2 and 3 show also that the nonthermal flux $I_{\mathrm{nt}}(t)$ (Figures 1(f), 2(f), and 3(f)) and the power $P_{\mathrm{wt}}(t)$ (Figures 1(i), 2(i), 3(i)) are correlated with the GOES time derivative (Figures 1(a), 2(a), and 3(a)).

\subsection{Goodness-of-fit}

The goodness of the spectral fits computed with the OSPEX code is specified with the $\chi^{2}$-criterion, based on the leastsquare difference between the theoretical spectral model (isothermal plus power-law nonthermal function) and the observed counts in the fitted energy range $\left[\varepsilon_{1}-\varepsilon_{2}\right]$. The fitted energy time interval (with a resolution of $1 \mathrm{keV}$ ) has about $n_{\text {bin }} \approx 30-10=20$ energy bins, while the model has four $\left(n_{\text {par }}=4\right)$ free parameters $\left(E M, T_{e}, A_{50}, \delta\right)$, yielding a degree of freedom $n_{\text {free }}=n_{\text {bin }}-n_{\text {par }} \approx 20-4=16$. In our spectral analysis of 191 flare events, we performed spectral fits, with an average of $n_{t} \approx 27$ time steps per event, amounting to a total of $N_{\text {spec }} \approx 191 \times 27=5157$ spectral fits. The values $\chi(t)$ of three events are shown in Figures 1(h), 2(h), and 3(h). The median values of these three events are $\chi=1.4,1.0$, and 1.3. We obtained in all 191 events a median goodness-of-fit value of $\chi<2$, after adjustment of the fitted energy range if necessary. The mean and standard deviations of the median $\chi^{2}$-values of all 191 events is $\chi=1.2 \pm 0.4$, which indicates that the fitted spectral model is adequate in the chosen fitted energy range. Of course, if one particular model, such as the two-component thermal-nonthermal model chosen here (Equation (7)), is found to be consistent with the data according to an acceptable goodness-of-fit criterion, it does not rule out alternative models. For instance, the thermal component is often modeled with an isothermal (singletemperature) spectrum, while a multithermal power-law function was found to fit the thermal flare component in most flares equally well (Aschwanden 2007).

\subsection{Temperature Definitions}

A representative value for the electron temperature during a flare can be defined in various ways. In Paper II, we measured the peak temperature $T_{\mathrm{AIA}}$ of the DEM distribution at the peak time of the flare, as well as the emission measure-weighted temperature $T_{w}$ (Equation (13) in Paper II), which approximately characterizes the "centroid" of the (logarithmic) DEM function. The mean ratio of these two temperature values was found to be $q_{T}=T_{\mathrm{AIA}} / T_{w}=0.31$ within a standard deviation by a factor of 2.0 (Figure 4, left panel). The emission measureweighted temperature $T_{w}$ is generally found to be higher, because near-symmetric DEM functions as a function of the logarithmic temperature are highly asymmetric on a linear temperature scale, with a centroid that is substantially higher than the logarithmic centroid.

On the other hand, spectral fits of RHESSI data with an isothermal component are known to have a strong bias toward the highest temperatures occurring in a flare, because the fitted energy range covers only the high-temperature tail of the DEM distribution function (Battaglia et al. 2005; Caspi et al. 2014; Ryan et al. 2014). A statistical study demonstrated that the high-temperature bias of RHESSI by fitting in the photon energy range of $\varepsilon \approx 6-12 \mathrm{keV}$ amounts to a factor of $T_{R} / T_{\mathrm{AIA}}=1.9 \pm 1.0$ (Ryan et al. 2014). Here we find that all RHESSI temperatures averaged during each flare are found in a range of $T_{R}=16-40 \mathrm{MK}$, which is about equal to the emission measure-weighted temperature, i.e., $T_{R} / T_{w}=0.90$ within a factor of 1.4 (Figure 4 , right panel). The $1 \sigma$ ranges (containing $67 \%$ of the values) of the various temperature definitions are $T_{\mathrm{AIA}} \approx 3-14 \mathrm{MK}, T_{w} \approx 20-30 \mathrm{MK}$, and $T_{R} \approx 19-28 \mathrm{MK}$. Thus, we should keep these different temperature definitions in mind when we calculate the lowenergy cutoff $e_{c}(t)$ as a function of the RHESSI temperature $T_{R}(t)$ (Equation (8) for the warm-target model).

The most decisive parameter in the determination of the nonthermal energy $E_{\mathrm{nt}}$ is the low-energy cutoff $e_{c}$ (Equation (4)), which is directly proportional to the temperature $T_{e}$ in the warm 

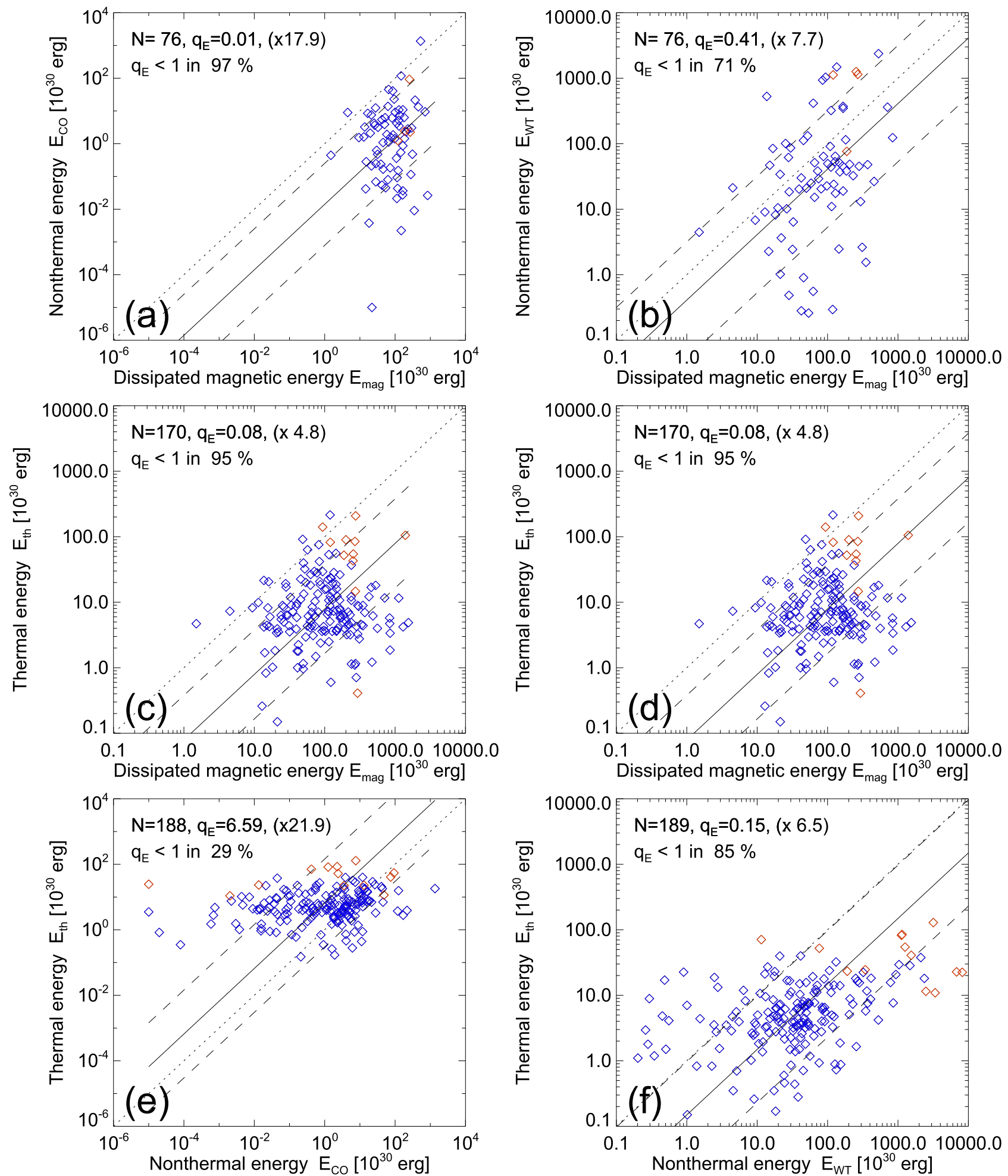

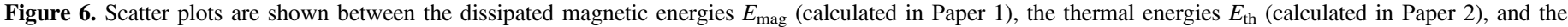

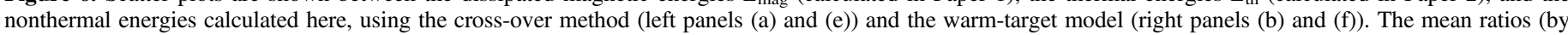

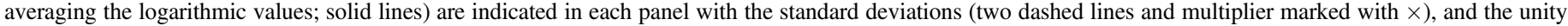
ratio (dotted line). The color code indicates X-class (red) and M-class flares (blue).

target (Equation (8)). The relevant temperature is a mixture of preflare plasma temperatures and upflowing evaporating flare plasma. In the absence of a sound model, we resort to the mean value of the DEM peak temperatures determined in flaring active regions, as determined with AIA in Paper II, yielding a mean value of $T_{\mathrm{AIA}}=8.8 \pm 6.0 \mathrm{MK}$ (Figure 4 left panel), averaged over $N=380 \mathrm{M}$ and X-class flare events. For the subset of 191 flare events observed with RHESSI, this mean value is $T_{e}=8.6 \mathrm{MK}$, or $k_{B} T_{e}=0.74 \mathrm{keV}$. Note that a deviation of the plasma temperature by a factor of two will result into a 


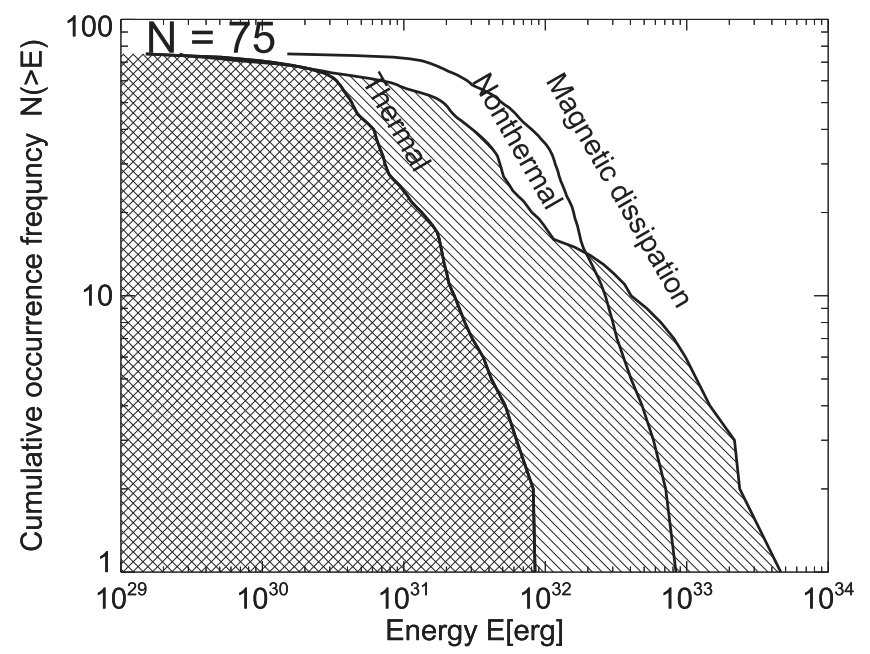

Figure 7. Cumulative occurrence frequency distributions of thermal, nonthermal, and dissipated magnetic energies in $75 \mathrm{M}$ and X-class flare events simultaneously observed with HMI, AIA, and RHESSI.

deviation in the determination of the nonthermal energy $E_{\mathrm{nt}}$ by about an order of magnitude (using a power law with a typical slope of $\gamma \approx 4$ in Equations (3) and (4).

\subsection{Nonthermal Energy Parameters}

The nonthermal energy in electrons, calculated as a time integral $E_{\mathrm{nt}}$ (Equation (4)), using the low-energy cutoff according to the warm thick-target model $e_{\mathrm{wt}}(t)$ (Section 2.3; Equation (8)), or alternatively the thermal/nonthermal crossover energy $e_{\mathrm{co}}(t)$ (Section 2.2), is the main objective of this study. Examples of the time evolution of the nonthermal parameters $\left[A(t), \delta(t), e_{\mathrm{co}}(t), e_{\mathrm{wt}}(t)\right]$ and the resulting nonthermal energies $d E_{\mathrm{nt}}(t)$ are shown in Figures 1-3. In Figure 5, we show statistical results of these parameters. Investigating the dependence of these parameters on the flare temperature $T_{R}$ we find that both the low-energy cutoff energy $e_{\mathrm{wt}}$ (Figure 5(a)) as well as the nonthermal (warm-target) energy $E_{\mathrm{nt}}$ (Figure 5(b)) are uncorrelated with the RHESSI temperature.

If we use the thermal-nonthermal cross-over method to estimate the low-energy cutoff, we find a systematically higher value, $e_{\mathrm{co}} \gtrsim e_{\mathrm{wt}}$ (Figure 5(c)). Consequently, the nonthermal energy estimated with the cross-over method is systematically lower than the nonthermal energy calculated with the warm-target model (Figure 5(d)). This result strongly depends on the assumption of the warm-target temperature. Based on a mean temperature of $T_{e}=8.6 \mathrm{MK}$ found in the active regions analyzed here, we derive low-energy cutoff energies of $e_{\mathrm{wt}}=6.2 \pm 1.6 \mathrm{keV}$ for the warmtarget model, which is significantly lower than the cross-over energies $e_{\mathrm{co}}=21 \pm 6 \mathrm{keV}$. If we adopt the warm-target model, we conclude that the cross-over method over-estimates the lowenergy cutoff and underestimates the nonthermal energies.

\subsection{Comparison of Magnetic, Nonthermal, and Thermal Energies}

In Figure 6, we show scatter plots of the nonthermal energy $E_{\mathrm{nt}}$ measured here with other forms of previously determined energies, such as the magnetic energy $E_{\text {mag }}$ (Paper I) and the (total pre-impulsive and post-impulsive) thermal energies $E_{\text {th }}$ (Paper II). The energy ratios are characterized with the means of the logarithmic energies in the following. The ratios between the three forms of energies are shown separately for the crossover method in the left-hand panels of Figure 6, and for the warm-target model in the right-hand panels of Figure 6.

The ratios between the nonthermal energies and the magnetically dissipated energy is $E_{\mathrm{co}} / E_{\mathrm{mag}}=0.01$ for the cross-over method, or $E_{\mathrm{wt}} / E_{\mathrm{mag}}=0.41$ for the warm-target model, respectively. Thus, the warm-target model yields ratios that are closer to unity, which is expected in terms of magnetic reconnection processes, where most of the magnetic energy is converted into particle acceleration. We find that the dissipated magnetic energy is sufficient to supply the energy in nonthermal particles in $71 \%$ for the warm-target model, or in 97\% for the cross-over model (Figures 6(a) and (b)).

The ratios between the thermal energies and the magnetically dissipated energy is $E_{\mathrm{th}} / E_{\mathrm{mag}}=0.08$ for both the cross-over or the warm-target model (Figures 6(c) and (d)). We find that the dissipated magnetic energy is sufficient to supply the thermal energy in $95 \%$.

Comparing the thermal with the nonthermal energies, we find a mean ratio of $E_{\mathrm{th}} / E_{\mathrm{wt}}=0.15$ for the warm-target model, or $E_{\mathrm{th}} / E_{\mathrm{co}}=6.46$ for the cross-over method. We find that the nonthermal energy is sufficient to supply the thermal energy in $85 \%$ for the warm-target model (Figure 6(f)), but only in $29 \%$ for the cross-over method. Thus, the warm-target model yields values that are closer to the expectations of the standard thicktarget model, where the thermal energy is entirely produced by the nonthermal energy of precipitating (nonthermal) electrons.

We show the comparison of nonthermal and thermal energies also in the form of cumulative size distributions in Figure 7, for the subset of 75 flares for which all three forms of energy (magnetic, thermal, nonthermal) could be calculated. We find that the nonthermal energy is typically an order of magnitude larger than the thermal energy in the statistical average. The nonthermal energy is smaller than the magnetic energy, as expected for magnetic reconnection processes, for smaller flares with energies of $E_{\mathrm{nt}}<3 \times 10^{32} \mathrm{erg}$. However, we find the opposite result for larger flares, with the nonthermal energy exceeding the magnetically dissipated energy, for large events with $E_{\mathrm{nt}}>3 \times 10^{32} \mathrm{erg}$. Since the uncertainties in nonthermal energies are about an order of magnitude and the dissipated magnetic energy exceeds the nonthermal energy in $71 \%$ (Figure 6(b)), we suspect that the largest nonthermal energies are overestimated, which would indicate that a higher value of the low-energy cutoff or a higher flare plasma temperature (than the mean active region temperature $T_{e}=8.6 \mathrm{MK}$ used here) could ameliorate the overestimated nonthermal energies.

We compare the occurrence frequency distributions of magnetic, nonthermal, and thermal energies, as well as those of the direct RHESSI observables: the peak counts $P$, total counts $C$, and durations $D$ (Figure 8 ). As a caveat, we have to be aware that these values for $P$ and $C$ are obtained from the online RHESSI flare catalog, and thus are not well-calibrated because they do not take attenuation or decimation into account. Nevertheless, taking these raw values, the magnetic and thermal energies have similar power-law slopes of $\alpha \approx 2.0$, while the nonthermal energies have a slightly flatter slope of $\alpha_{\mathrm{nt}}=1.41 \pm 0.10$, which can be compared with a previous study, where a power-law slope of $\alpha_{\mathrm{nt}}=1.53 \pm 0.02$ was found (Crosby et al. 1993). The latter study is actually based on larger statistics, containing 2878 flare events observed 

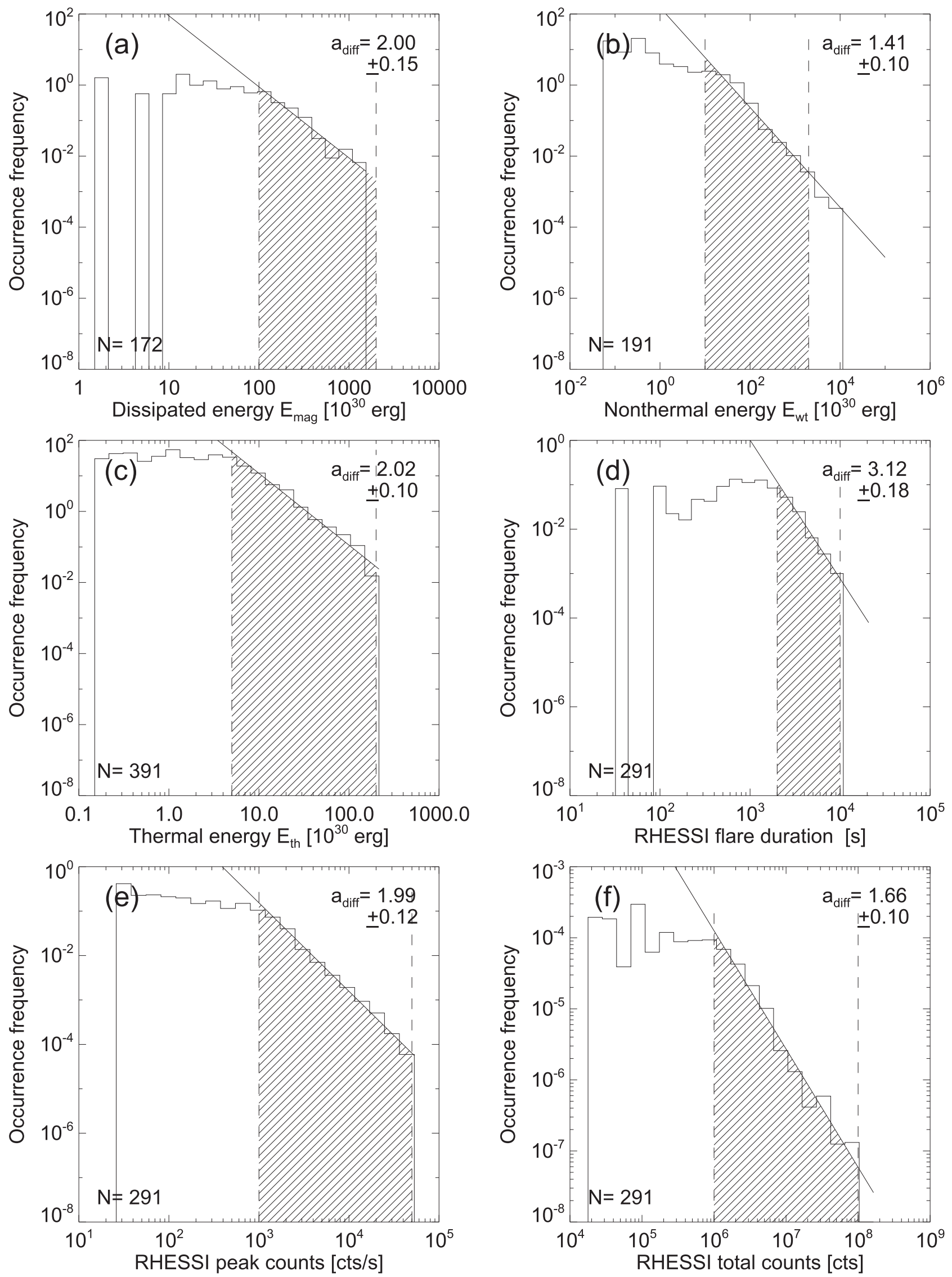

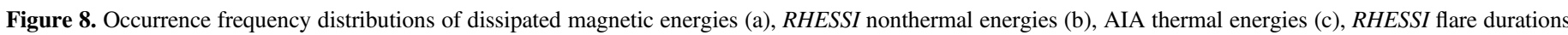

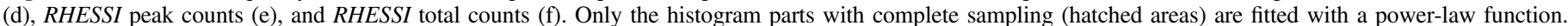


with HXRBS/SMM during 1980-1982 (Crosby et al. 1993), but with a higher assumed low-energy cutoff of $e_{c}>25 \mathrm{keV}$.

\section{DISCUSSION}

\subsection{Energy Partition in Flares}

While we determined the dissipated magnetic energies $E_{\text {mag }}$ (Paper I; called $E_{\text {diss }}$ therein), thermal energies $E_{\text {th }}$ (Paper II), and the nonthermal energies $E_{\mathrm{nt}}$, we can ask now the question how the energy partition from primary to secondary energy dissipation works in solar flares. Many solar flare models are based on a magnetic reconnection process, where a stressed non-potential magnetic field becomes unstable and undergoes a reconfiguration toward a lower magnetic energy state, releasing during this process some amount $E_{\text {mag }}=q_{\text {diss }} E_{\text {free }}$ of the magnetic free energy $E_{\text {free }}$ (defined by the difference between the non-potential and the potential energy, $E_{\text {free }}=E_{\mathrm{np}}-E_{p}$ ). Excluding alternative energy sources, we hypothesize that this dissipated magnetic energy $E_{\mathrm{mag}}$ is considered to be the entire available primary energy input, while other energy conversion processes represent secondary steps that need to add up in the energy budget,

$$
E_{\mathrm{mag}}=\left(E_{\mathrm{nt}}+E_{\mathrm{cme}}+\ldots\right)>E_{\mathrm{nt}},
$$

such as the nonthermal energy $E_{\mathrm{nt}}$ that goes into acceleration of particles, or the energy $E_{\mathrm{cme}}$ to accelerate an accompanying CME. The nonthermal energy $E_{\mathrm{nt}}$ may be further subdivided into energies in electrons $E_{\mathrm{nt}, \mathrm{e}}$ and ions $E_{\mathrm{nt}, \mathrm{i}}$,

$$
E_{\mathrm{nt}}=\left(E_{\mathrm{nt}, \mathrm{e}}+E_{\mathrm{nt}, \mathrm{i}}+\ldots\right)>E_{\mathrm{nt}, \mathrm{e}}
$$

while the CME energy $E_{\text {cme }}$ consists of the kinetic energy $E_{\text {kin }}$ and the gravitational potential energy $E_{\text {grav }}$, and part of it may be converted into acceleration of particles in the interplanetary CME shock $\left(E_{\mathrm{nt}, \mathrm{cme}}\right)$, which are particularly present in solar energetic particle events,

$$
E_{\mathrm{cme}}=E_{\mathrm{kin}}+E_{\mathrm{grav}}+E_{\mathrm{nt}, \mathrm{cme}}+\ldots
$$

We have to be careful to avoid double-counting secondary energies, because there may be some tertiary energy conversion processes, such as heating of chromospheric plasma according to the thick-target bremsstrahlung model, $E_{\mathrm{th}}$, while upgoing nonthermal particles escape into interplanetary space, carrying an energy of $E_{\text {nt,esc }}$,

$$
E_{\mathrm{nt}}=\left(E_{\mathrm{th}}+E_{\mathrm{nt}, \mathrm{esc}}+\ldots\right)>E_{\mathrm{th}} .
$$

Since we have measured only three types of energies so far, $E_{\mathrm{mag}}, E_{\mathrm{nt}}$, and $E_{\mathrm{nt}}$, we can only test the inequalities given on the right-hand-side of Equations (9) and (12) at this point.

Based on the nonthermal energies in electrons determined in this work, we can answer the question whether the so far measured magnetic energy is sufficient to accelerate the electrons observed in hard X-rays, i.e., $E_{\text {mag }}>E_{\mathrm{nt}}$, as expected for magnetic reconnection models. Relying on the warm-target model we found that $41 \%$ of the dissipated magnetic energy (with a standard deviation of about an order of magnitude) is converted into acceleration of nonthermal electrons, or a total amount of $\approx 82 \%$ for both electrons and ions in the case of equipartition, while the rest is available to accelerate CMEs. There are few statistical estimates of the flare energy budget in the literature (besides the work of Emslie et al. 2012; Warmuth \& Mann 2016). One early study quoted that the nonthermal energy in electrons $>20 \mathrm{keV}$ contains $10 \%-50 \%$ of the total energy output for the 1972 August flares (Lin \& Hudson 1976; Hudson \& Ryan 1995), which is consistent with our result of $41 \%$ within the measurement uncertainties.

Comparing the energy ranges determined in this global flare energetics project with those obtained from 38 events in Emslie et al. (2012), we find higher amounts of nonthermal flare electron energies in the statistical average, covering the range of $E_{\mathrm{nt}} \approx(20-2000) \times 10^{30} \mathrm{erg}$ (Figure 9), which is mostly accounted for by a lower value of the low-energy cutoff predicted by the warm-target model (Kontar et al. 2015) for some events, while cutoff energies with the highest acceptable value of the $\chi^{2}$ were used in Emslie et al. (2012). The magnetically dissipated energies appear to be overestimated by an order of magnitude (Figure 9) in Emslie et al. (2012), based on the ad hoc assumption that the dissipated energy amounts to $30 \%$ of the potential field energy therein (Paper I). On the other hand, the thermal energies appear to be underestimated by at least an order of magnitude (Figure 9) in Emslie et al. (2012) due to the isothermal approximation, as discussed in Paper II.

\subsection{Insufficiency of the Thick-target Model?}

A second question we can answer is whether the nonthermal energy in electrons is sufficient to heat the flare plasma by the chromospheric evaporation process, as expected in the thicktarget model according to the Neupert effect (Dennis \& Zarro 1993), which requires $E_{\mathrm{nt}}>E_{\mathrm{th}}$. Based on the warmtarget model, we found a mean (logarithmic) ratio of $E_{\mathrm{th}}=0.15 E_{\mathrm{nt}}$ (Figure 6(f)). The fraction of flares that have a thermal energy less than the nonthermal energy, as expected in the standard thick-target model, amounts in our analysis to $\approx 85 \%$ for the warm-target method, or $\approx 29 \%$ for the cross-over model.

This means that the thick-target model could be insufficient to supply enough energy to explain the thermal energy produced by the chromospheric evaporation process in about $15 \%$ of the flares for the warm-target model, or in $71 \%$ for the cross-over model. Thus, the cross-over model would pose a series problem for the thick-target model. The insufficiency of the thick-target model has been addressed as a failure of the theoretical Neupert effect (Veronig et al. 2005; Warmuth \& Mann 2016), which invokes testing of the correlation between the electron beam power (from RHESSI) and the time derivative of the thermal energy heating rate (from GOES). From such studies, it was concluded that (1) fast electrons are not the main source of soft X-ray plasma supply and heating, (2) the beam low cutoff energy varies with time, or (3) the theoretical Neupert effect is strongly affected by the source geometry (Veronig et al. 2005). If the thermally dominated flares cannot be fully explained by the thick-target model, additional heating sources besides precipitating electrons would be required. The most popular alternative to the thicktarget model is heating by thermal conduction fronts (Brown et al. 1979; Emslie \& Brown 1980; Smith \& Brown 1980; Smith \& Harmony 1982; Batchelor et al. 1985; Reep et al. 2016). Other forms of direct heating (for an overview see chapter 16 in Aschwanden 2004) occur via (1) resistive or Joule heating processes, such as anomalous resistivity heating (Duijveman et al. 1981; Holman 1985; Tsuneta 1985), ionacoustic waves (Rosner et al. 1978a), electron ion-cyclotron waves (Hinata 1980), (2) slow-shock heating (Cargill \& Priest 1983; Hick \& Priest 1989), (3) electron beam heating 


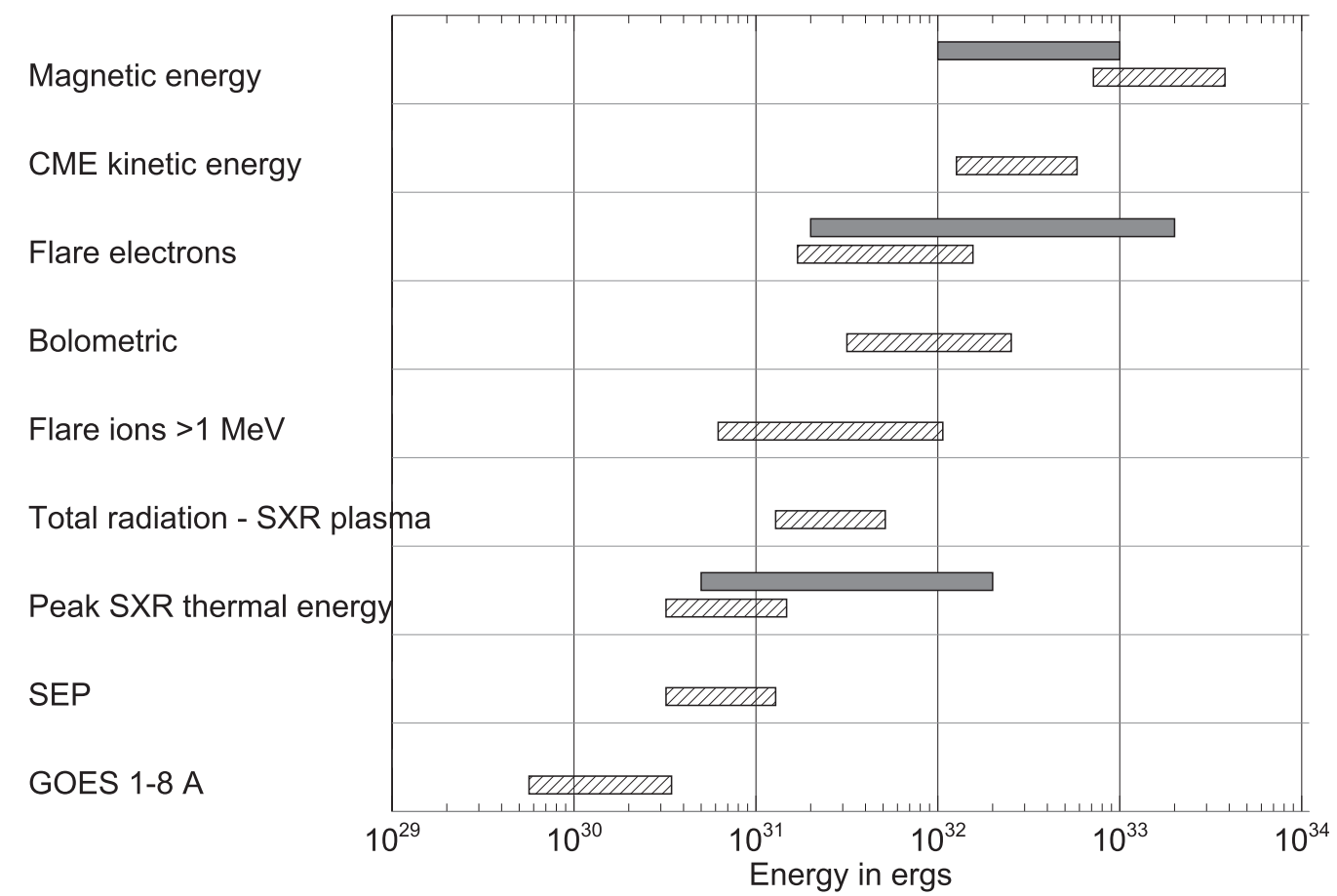

Figure 9. Bar chart showing the logarithmic ranges of energy distributions according to the study of 38 events in Emslie et al. (2012; hatched boxes). For comparison, the magnetic energies in 172 events (Aschwanden et al. 2014), the thermal energies in 391 events (Aschwanden et al. 2015), and the nonthermal energies in this study here are shown (all in gray boxes). The gray boxes exclude incompletely sampled ranges.

by Coulomb collisional loss in the corona (Fletcher 1995, 1996; Fletcher \& Martens 1998), (4) proton beam heating by kinetic Alfvén waves (Voitenko 1995, 1996), or (5) inductive current heating (Melrose 1995, 1997).

The thick-target model fails to explain the observed amount of thermal energy only in a small number of flares for the warm-target model, while it is a larger number of events for the cross-over method. However, it is more likely that the crossover method overestimates the low-energy cutoff, which underestimates the nonthermal energies, while the physicsbased warm-target model leads to higher nonthermal energies, in which case the problem with the insufficiency of the thicktarget model goes away.

\subsection{Nonthermal Low-energy Cutoff in Flares}

We outlined two different methods to infer a low-energy cutoff. The first method consists of measuring the cross-over between the fitted thermal and nonthermal spectral components, which yields an upper limit on the low-energy cutoff, but a statistical test demonstrates that the obtained values $\left(e_{\mathrm{co}}=21 \pm 6 \mathrm{keV}\right)$ are significantly higher than those obtained from the warm-target model $\left(e_{\mathrm{wt}}=6.2 \pm 1.6 \mathrm{keV}\right)$. There are pros and cons for each method. The cross-over method requires a dominant thermal component, which is not always detectable in the spectrum, in which case the cross-over energy has a large uncertainty. The warm-target model requires the measurement of the (warm) flare temperature, which is measured at lower values from DEMs at EUV wavelengths than from hard X-ray spectra observed with RHESSI. Moreover, the spatial temperature distribution is very inhomogeneous and the location with the dominant temperature component relevant for the warm-target collisional energy loss may be a mixture of colder preflare plasma in active regions and heated evaporating flare plasma at the location of instantaneous electron precipitation. In summary, the value of the low-energy cutoff is strongly dependent on the assumed warm-target temperature, for which no physical model is established yet.

In this study, we also investigated the temporal evolution of the low-energy cutoff $e_{c}(t)$, for instance, as shown in Figures 1(j), 2(j), and 3(j), but we do not recognize a systematic pattern indicating how the evolution of this low-energy cutoff is related to other flare parameters.

\section{CONCLUSIONS}

The energy partition study of Emslie et al. (2012) was restricted to 38 large solar eruptive events. In a more comprehensive study of the global flare energetics we choose a data set that contains the 400 largest (GOES M- and X-class) flare events observed during the first 3.5 years of the $S D O$ era. Previously, we determined the dissipated magnetic energies $E_{\text {mag }}$ in these flares based on fitting the vertical-current approximation of a nonlinear force-free field (NLFFF) solution to the loop geometries detected in EUV images from SDO/ AIA, a new method that could be applied to 177 events with a heliographic longitude of $\leqslant 45^{\circ}$ (Paper I). We also determined the thermal energy $E_{\mathrm{th}}$ in the soft X-ray and EUV-emitting plasma during the flare peak times based on a multitemperature DEM forward-fitting method to SDO/AIA image pixels with spatial synthesis, which was applicable to 391 events (Paper II). In the present study, we determined the nonthermal energy $E_{\mathrm{nt}}$ contained in accelerated electrons based on spectral fits to RHESSI data using the OSPEX software, which was applicable to 191 events. The major conclusions of the new results emerging from this study are as follows.

1. The (logarithmic) mean energy ratio of the nonthermal energy to the total magnetically dissipated flare energy is 
found to be $E_{\mathrm{nt}} / E_{\mathrm{mag}}=0.41$, with a logarithmic standard deviation corresponding to a factor of $\approx 8$, which yields an uncertainty of $\sigma / \sqrt{N}=0.41 / \sqrt{191}=0.03$ for the mean, i.e., $E_{\mathrm{nt}} / E_{\mathrm{mag}}=0.41 \pm 0.03$. The majority $(\approx 85 \%)$ of the flare events fulfill the inequality $E_{\mathrm{nt}} / E_{\mathrm{mag}}<1$, which suggests that magnetic energy dissipation (most likely by a magnetic reconnection process) provides sufficient energy to accelerate the nonthermal electrons detected by bremsstrahlung in hard $\mathrm{X}$-rays. Our results yield an order of magnitude higher electron acceleration efficiency than previous estimates, i.e., $E_{\mathrm{nt}} / E_{\mathrm{mag}}=0.03 \pm 0.005$ (with $N=37$, Emslie et al. 2012).

2. The (logarithmic) mean of the thermal energy $E_{\mathrm{th}}$ to the nonthermal energy $E_{\mathrm{nt}}$ is found to be $E_{\mathrm{th}} / E_{\mathrm{nt}}=0.15$, with a logarithmic standard deviation corresponding to a factor of $\approx 7$. The fraction of flares with thermal energy smaller than the nonthermal energy, as expected in the thick-target model, is found to be the case for $\approx 85 \%$ only. Therefore, the thick-target model is sufficient to explain the full amount of thermal energy in most flares, in the framework of the warm-target model. The cross-over method shows the opposite tendency, but we suspect that the cross-over method overestimates the low-energy cutoff and underestimates the nonthermal energies. Previous estimates yielded a similar ratio, i.e., $E_{\mathrm{th}} / E_{\mathrm{nt}}=$ 0.15 (Emslie et al. 2012).

3. A corollary of the two previous conclusions is that the thermal to magnetic energy ratio is $E_{\text {th }} / E_{\text {mag }}=0.08$. A total of $95 \%$ of flares fulfills the inequality $E_{\mathrm{nt}} / E_{\mathrm{mag}}<1$, indicating that all thermal energy in flares is supplied by magnetic energy. Previous estimates were a factor of 17 lower, i.e., $E_{\mathrm{th}} / E_{\mathrm{mag}}=0.0045$ (Emslie et al. 2012), which would imply a very inefficient magnetic to thermal energy conversion process.

4. The largest uncertainty in the calculation of nonthermal energies, the low-energy cutoff, is found to yield different values for two used methods, i.e., $e_{\mathrm{wt}}=6.2 \pm 1.6 \mathrm{keV}$ for the warm thick-target model, versus $e_{\mathrm{co}}=21 \pm 6 \mathrm{keV}$ for the thermal/nonthermal cross-over method. The calculation of the nonthermal energies is highly sensitive to the value of the low-energy cutoff, which strongly depends on the assumed (warm-target) temperature.

5. The flare temperature can be characterized with three different definitions, for which we found the following (67\%-standard deviation) ranges: $T_{\mathrm{AIA}} \approx 3-14 \mathrm{MK}$ for the AIA DEM peak temperature, $T_{w} \approx 20-30 \mathrm{MK}$ for the emission measure-weighted temperatures, and $T_{R} \approx 17-36 \mathrm{MK}$ for the RHESSI high-temperature DEM tails. The median ratios are found to be $T_{\mathrm{AIA}} / T_{w}=0.31$ and $T_{R} / T_{w}=0.90$. The mean active region temperature evaluated from DEMs with AIA, $T_{e}=8.6 \mathrm{MK}$, is used to estimate the low-energy cutoff $e_{c}$ of the nonthermal component according to the warm-target model, i.e., $e_{c} \approx \delta\left(k_{B} T_{R}\right)$. The low-energy cutoff $e_{c}$ of the nonthermal spectrum has a strong functional dependence on the temperature $T_{R}$.

In summary, our measurements appear to confirm that the magnetically dissipated energy is sufficient to explain thermal and nonthermal energies in solar flares, which strongly supports the view that magnetic reconnection processes are the primary energy source of flares. The nonthermal energy, which represents the primary energy source of the thick-target model, is sufficient to explain the full amount of thermal energies in $71 \%$ of the flares, according to the novel warmtarget model (Kontar et al. 2011). However, the derived nonthermal energies are highly dependent on the the assumed temperature in the warm-target plasma, for which a sound physical model should be developed (see for instance Appendices B and C), before it becomes a useful tool to estimate the low-energy cutoff of nonthermal energy spectra. Future studies of this global flare energetics project may also quantify additional forms of energies, such as the kinetic energy in CMEs, and radiated energies in soft X-rays, EUV, and white-light (bolometric luminosity).

We acknowledge useful comments from an anonymous referee and discussions with Brian Dennis, Gordon Emslie, Iain Hannah, Ryan Milligan, Linhui Sui, Daniel Ryan, Richard Schwartz, Alexander Warmuth, and software support from Kim Tolbert and Samuel Freeland. This work was partially supported by NASA contract NAS5-98033 of the RHESSI mission through University of California, Berkeley (subcontract SA2241-26308PG), and by NASA contract NNG 04EA00C of the SDO/AIA instrument. A.C. and J.M.M. were also supported by NASA grant NNX15AK26G.

\section{APPENDIX A COLLISIONAL TIME-OF-FLIGHT MODEL}

We can derive a collisional time-of-flight model for the thermal/nonthermal cross-over energy that is complementary to the warm-target model of Kontar et al. (2015). For stochastic acceleration models, where particles gain and lose energy randomly, the collisional deflection time yields an upper time limit during which a particle can be efficiently accelerated. The balance between acceleration and collisions can lead to the formation of a kappa-distribution according to some solar flare models (Bian et al. 2014). For solar flares, we can thus estimate the cross-over energy between collisional and collisionless electrons by setting the collisional deflection time $t_{\text {defl, }}$

$$
t_{\mathrm{defl}} \approx 0.95 \times 10^{8}\left(\frac{e_{\mathrm{keV}}^{3 / 2}}{n_{e}}\right)\left(\frac{20}{\ln \Lambda}\right),
$$

where $\ln \Lambda \approx 20$ is the Coulomb logarithm, equal to the (relativistic) time-of-flight propagation time between the coronal acceleration site and the chromospheric thick-target energy loss site,

$$
t_{\mathrm{TOF}}=\frac{L_{\mathrm{TOF}}}{v}=\frac{L_{\mathrm{TOF}}}{\beta c},
$$

where the relativistic speed $\beta=v / c$,

$$
\beta=\sqrt{1-\frac{1}{\gamma^{2}}}
$$

is related to the kinetic energy $e_{\text {kin }}$ of the electron by

$$
e_{\mathrm{kin}}=m_{e} c^{2}(\gamma-1)=511(\gamma-1)[\mathrm{keV}],
$$

where $\gamma$ represents here the relativistic Lorentz factor (not to be confused with the spectral slope of the photon spectrum used above, i.e., Equation (1)). So, setting these two timescales 
equal,

$$
t_{\mathrm{defl}}=t_{\mathrm{TOF}}
$$

yields the relationship, using $\ln \Lambda \approx 20$,

$$
(\gamma-1)^{3 / 2}\left(1-\frac{1}{\gamma^{2}}\right)^{1 / 2}=\frac{L_{\mathrm{TOF}} n_{e}}{0.95 \times 10^{8} \times 511^{3 / 2} c} .
$$

Using the low-relativistic approximation (for $\gamma \gtrsim 1$ ),

$$
\begin{aligned}
(\gamma-1)^{3 / 2}\left(1-\frac{1}{\gamma^{2}}\right)^{1 / 2} & =(\gamma-1)^{3 / 2} \frac{(\gamma-1)^{1 / 2}(\gamma+1)^{1 / 2}}{\gamma} \\
& =\frac{(\gamma-1)^{2}(\gamma+1)^{1 / 2}}{\gamma} \\
& \approx(\gamma-1)^{2} \sqrt{2}
\end{aligned}
$$

we obtain,

$$
(\gamma-1)^{2} \sqrt{2} \approx 0.003 \times\left(\frac{L_{\mathrm{TOF}}}{10^{9} \mathrm{~cm}}\right)\left(\frac{n_{e}}{10^{11} \mathrm{~cm}^{-3}}\right) \quad[\mathrm{keV}] .
$$

and by inserting $(\gamma-1)=e_{c} / 511 \mathrm{keV}$ from Equation (16), we find the cross-over energy $e_{c} \approx e_{\text {kin }}$ can be explicitly expressed as

$$
e_{c} \approx 24\left(\frac{L_{\mathrm{TOF}}}{10^{9} \mathrm{~cm}}\right)^{1 / 2}\left(\frac{n_{e}}{10^{11} \mathrm{~cm}^{-3}}\right)^{1 / 2} \quad[\mathrm{keV}]
$$

This expression requires the measurement of a mean length scale $L_{\mathrm{TOF}}$ of flare loops and an average electron density $n_{e}$ where electrons propagate.

Turning the argument around predicts a time-of-flight distance $L_{\mathrm{TOF}} \propto e_{c}^{2} / n_{e}$ as a function of the low-energy cutoff $e_{c}$, which is a similar concept that has been applied to model the size $L$ of the acceleration region as a function of the electron energy $e$, i.e., $\left(L-L_{0}\right) \propto e^{2} / n_{e} \quad$ (Guo et al. 2012a, 2012b, 2013; Xu et al. 2008).

\section{APPENDIX B}

\section{THE ROSNER-TUCKER-VAIANA MODEL}

At the peak time of a flare, an energy balance between plasma heating and cooling occurs at the turnover point of the temperature maximum (Aschwanden \& Tsiklauri 2009), which corresponds to the scaling law of Rosner et al. (1978b) that was originally applied to steady-state heating of coronal loops, where an energy balance between the heating rate and the conductive and radiative cooling time is assumed. The RTV scaling law, $T^{3} \propto p L$, can be expressed in terms of the ideal gas pressure $p=3 n_{e} k_{B} T$, which yields for the loop apex temperature $T_{\mathrm{RTV}}$,

$$
T_{\mathrm{RTV}}=0.0011\left(n_{e} L_{\mathrm{RTV}}\right)^{1 / 2} .
$$

The loop half length and time-of-flight distance scale approximately with the flare size, $L_{\mathrm{TOF}} \approx L_{\mathrm{RTV}} \approx L$. Interestingly, the parameter combination $\left(n_{e} L\right)^{1 / 2}$ occurs also in the expression for the collisional low-energy cutoff (Equation (21)), so that we can insert the RTV scaling law and obtain an expression for the low-energy cutoff energy $e_{c}$ that depends on the temperature $T_{\mathrm{RTV}}$ only,

$$
e_{c} \approx 25\left(k_{B} T_{\mathrm{RTV}}\right) \quad[\mathrm{keV}],
$$

which is similar to the result of the warm-target model (Equation (8)). However, while the warm-target model is applied to the evaporating upflowing flare plasma, which has temperatures of $T_{e} \approx 10-25 \mathrm{MK}$, the collisional deflection model should be applied to the temperature of the cooler preflare loops, where the accelerated particles propagate from the acceleration site to the thick-target site. These cooler preflare loops may have typical coronal temperatures of $T_{\mathrm{RTV}} \approx 5-6 \mathrm{MK}(\approx 0.43-0.52 \mathrm{keV})$ in active regions (Hara et al. 1992), which predicts low-energy cutoff energies of $e_{c}=11-13 \mathrm{keV}$. If the time-of-flight distance $L_{\mathrm{TOF}}$ is corrected for magnetic twist and the pitch angle of the electrons, the effective time-of-flight distance is about $L_{\mathrm{TOF}} \lesssim 2 L$ (Aschwanden et al. 1996), which increases the low-energy cutoff energy by a factor of $\sqrt{2}$, predicting values of $e_{c}=15-18 \mathrm{keV}$. Combining Equations (8) and (23), the RTV model predicts a relationship between the preflare temperature $T_{\text {pre }}=T_{\mathrm{RTV}}$ and the (maximum) flare temperature $T_{\text {flare }}$,

$$
T_{\text {pre }} \approx T_{\text {flare }}\left(\frac{\delta}{25}\right)
$$

which yields $T_{\text {pre }} \approx(0.12-0.24) T_{\text {flare }}$ for a range of spectral slopes $\delta \approx 3-6$. Given the fact that flare temperatures are typically found in the range of $T_{\text {flare }} \approx 10-25 \mathrm{MK}$, while preflare temperatures amount to typical coronal temperatures in active regions, $T_{\text {pre }} \approx 1-4 \mathrm{MK}$, we would expect indeed temperature ratios of $T_{\text {pre }} / T_{\text {flare }} \approx 0.1-0.16$.

\section{APPENDIX C \\ THE RUNAWAY ACCELERATION MODEL}

Some particle acceleration models involve DC electric fields that accelerate electrons and ions out of the bulk plasma. Since the frictional drag on the electrons decreases with increasing particle velocity $\left(\nu \propto v^{-3}\right)$, electrons in the initial thermal distribution with a high enough velocity will not be confined to the bulk current, but will be freely accelerated out of the thermal distribution (Kuijpers et al. 1981; Holman 1985), a process that is called runaway acceleration. A thermal electron of velocity $v_{e}$ will run away if the electric field strength is greater than the Dreicer field $E_{D}$,

$$
E_{D}=\frac{m}{e} v_{e} \nu_{e}
$$

where $m$ is the electron mass, $e$ is the electron charge, $v_{e}$ is the electron velocity, and $\nu_{e}$ is the electron collision frequency. Since the square of the (non-relativistic) speed $v_{e}$ scales with the kinetic energy, $E_{\text {kin }}=(1 / 2) m_{e} v_{e}^{2}$, the critical runaway energy $E_{\text {ra }}$ can be characterized by the ratio of the critical velocity $v_{e}$ to the thermal speed $v_{\mathrm{th}}$,

$$
E_{\mathrm{ra}}=E_{\mathrm{th}}\left(\frac{v_{e}}{v_{\mathrm{th}}}\right)^{2},
$$

We can associate this critical runaway energy $E_{\text {ra }}$ with the lowenergy cutoff $e_{c}$ and obtain again a relationship that scales with 
the plasma temperature $T_{e}$ for a given critical velocity ratio,

$$
e_{c} \approx E_{\mathrm{ra}}=k_{B} T_{e}\left(\frac{v_{e}}{v_{\mathrm{th}}}\right)^{2} \quad[\mathrm{keV}] .
$$

Thus, for a typical velocity ratio of $\left(v_{e} / v_{\text {th }}\right) \approx 2-3$ and a plasma temperature range of $T_{e} \approx 5-6 \mathrm{MK} \approx 0.43-0.52 \mathrm{keV}$ in active regions, this model predicts a range of $e_{c} \approx 1.7-8.3 \mathrm{keV}$. Combining the relationships of the warm-target model (Equation (8)) and the runaway acceleration model (Equation (25)) yields then a prediction for the nonthermal speed ratio of the runaway electrons,

$$
\left(\frac{v_{e}}{v_{\mathrm{th}}}\right) \approx \sqrt{\delta} \approx(1.7-2.4),
$$

which is consistent with solar parameters used in runway models (Kuijpers et al. 1981; Holman 1985). Implications of runway acceleration models for sub-Dreicer and super-Dreicer fields are also discussed in Guo et al. (2013) and Miller et al. (1997).

\section{REFERENCES}

Aschwanden, M. J. 2004, Physics of the Solar Corona-An Introduction (New York: Springer)

Aschwanden, M. J. 2007, ApJ, 661, 1242

Aschwanden, M. J. 2013, SoPh, 287, 323

Aschwanden, M. J. 2016, ApJSS, 224, 25

Aschwanden, M. J., Boerner, P., Ryan, D., et al. 2015, ApJ, 802, 53

Aschwanden, M. J., Bynum, R. M., Kosugi, T., Hudson, H. S., \& Schwartz, R. A. 1997, ApJ, 487, 936

Aschwanden, M. J., Kosugi, T., Hudson, H. S., Wills, M. J., \& Schwartz, R. A 1996, ApJ, 470, 1198

Aschwanden, M. J., \& Tsiklauri, D. 2009, ApJS, 185, 171

Aschwanden, M. J., Xu, Y., \& Jing, J. 2014, ApJ, 797, 50

Batchelor, D. A., Crannell, C. J., Wiehl, H. J., \& Magun, A. 1985, ApJ, 295, 258

Battaglia, M., Grigis, P. C., \& Benz, A. O. 2005, A\&A, 439, 737

Bian, N. H., Emslie, A. G., Stackhouse, D. J., \& Kontar, E. P. 2014, ApJ, 796, 142

Brown, J. C. 1971, SoPh, 18, 489

Brown, J. C. 1974, in Proc. Symp. IAU Coll. 57, Coronal Disturbances, ed. G. J. Newkirk, Jr. (Dordrecht: Reidel), 523

Brown, J. C., Spicer, D. S., \& Melrose, D. B. 1979, ApJ, 228, 592

Cargill, P. J., \& Priest, E. R. 1983, ApJ, 266, 383

Caspi, A. 2010, PhD Thesis, Univ. California, Berkeley

Caspi, A., Krucker, S., \& Lin, R. P. 2014, ApJ, 781, 43

Caspi, A., \& Lin, R. P. 2010, ApJ, 718, 1476

Christe, S., Hannah, I. G., Krucker, S., McTiernan, J., \& Lin, R. P. 2008, ApJ, 677, 1385

Crosby, N. B., Aschwanden, M. J., \& Dennis, B. R. 1993, SoPh, 143, 275

Dennis, B. R. 1985, SoPh, 100, 465

Dennis, B. R., \& Zarro, D. M. 1993, SoPh, 146, 177
Duijveman, A., Hoyng, P., \& Ionson, J. A. 1981, ApJ, 245, 721

Dulk, G. A., \& Dennis, B. R. 1982, ApJ, 260, 875

Emslie, A. G., \& Brown, J. C. 1980, SoPh, 237, 1015

Emslie, A. G., Dennis, B. R., Shih, A. Y., et al. 2012, ApJ, 759, 71

Fletcher, L. 1995, A\&A, 303, L9

Fletcher, L. 1996, A\&A, 310, 661

Fletcher, L., \& Martens, P. C. H. 1998, ApJ, 505, 418

Galloway, R. K., MacKinnon, A. L., Kontar, E. P., \& Helander, P. 2005, A\&A, 438, 1107

Goncharov, P. R., KJuteev, B. V., Ozaki, T., \& Sudo, S. 2010, PhPl, 17 , 112313

Guo, J., Emslie, A. G., Kontar, E. P., et al. 2012a, A\&A, 543, A53

Guo, J., Emslie, A. G., Massone, A. M., Piana, M., \& Piana, M. 2012b, ApJ, 755,32

Guo, J., Emslie, A. G., \& Piana, M. 2013, ApJ, 766, 28

Hannah, I. G., Christe, S., Krucker, S., et al. 2008, ApJ, 677, 704

Hannah, I. G., Kontar, E. P., \& Sirenko, O. K. 2009, ApJL, 707, L45

Hara, H., Tsuneta, S., Lemen, J. R., Acton, L. W., \& McTiernan, J. M. 1992, PASJ, 44, L135

Hick, P., \& Priest, E. R. 1989, SoPh, 122, 111

Hinata, S. 1980, ApJ, 235, 258

Holman, G. D. 1985, ApJ, 293, 584

Holman, G. D. 2003, ApJ, 586, 606

Holman, G. D. 2012, ApJ, 745, 52

Holman, G. D., Aschwanden, M. J., Aurass, H., et al. 2011, SSRv, 159, 107

Holman, G. D., Sui, L., Schwartz, R. A., \& Emslie, A. G. 2003, ApJ, 595, L97

Hudson, H., \& Ryan, J. 1995, ARA\&A, 33, 239

Jeffrey, N. L. S., Kontar, E. P., Bian, N. H., \& Emslie, A. G. 2014, ApJ, 787, 86

Kasparova, J., Karlicky, M., Kontar, E. P., \& Dennis, B. R. 2005, Sol. Phys., 232, 63

Kontar, E. P., \& Brown, J. C. 2006, AdSpR, 38, 945

Kontar, E. P., Brown, J. C., \& Emslie, A. G. 2011, SSRv, 159, 301

Kontar, E. P., Dickson, E., \& Kasparova, J. 2008, SoPh, 252, 139

Kontar, E. P., Jeffrey, N. L. S., Emslie, A. G., \& Bian, N. H. 2015, ApJ, 809,35

Kuijpers, J., Van Der Post, P., \& Slottje, C. 1981, A\&A, 103, 331

Lin, R. P., Dennis, B. R., Jurford, G. J., et al. 2002, SoPh, 210, 3

Lin, R. P., \& Hudson, H. S. 1976, SoPh, 50, 153

Melrose, D. B. 1995, ApJ, 451, 391

Melrose, D. B. 1997, ApJ, 486, 521

Miller, J. A., Cargill, P. J., Emslie, A. G., et al. 1997, JGR, 102, 14631

Reep, J. W., Bradshaw, S. J., \& Holman, G. D. 2016, ApJ, 818, 44

Rosner, R., Golub, L., Coppi, B., \& Vaiana, G. S. 1978a, ApJ, 220, 643

Rosner, R., Tucker, W. H., \& Vaiana, G. S. 1978b, ApJ, 220, 643

Ryan, D. F., O'Flannagain, A. M., Aschwanden, M. J., \& Gallagher, P. T. 2014, SoPh, 289, 2547

Smith, D. F., \& Brown, J. C. 1980, ApJ, 242, 799

Smith, D. F., \& Harmony, D. W. 1982, ApJ, 252, 800

Su, Y., Holman, G. D., \& Dennis, B. R. 2011, ApJ, 731, 106

Sui, L., Holman, G. D., \& Dennis, B. R. 2007, ApJ, 670, 862

Tsuneta, S. 1985, ApJ, 290, 353

Veronig, A. M., Brown, J. C., Dennis, B. R., et al. 2005, ApJ, 621, 482

Voitenko, Y. M. 1995, SoPh, 161, 197

Voitenko, Y. M. 1996, SoPh, 168, 219

Warmuth, A., Holman, G. D., Dennis, B. R., et al. 2009, ApJ, 699, 917

Warmuth, A., \& Mann, G. 2016, A\&A, 588, A115

Xu, Y., Emslie, A. G., \& Hurford, G. J. 2008, ApJ, 673, 576 